\title{
Hamiltonian thermodynamics of charged three-dimensional dilatonic black holes
}

\author{
Gonçalo A. S. Dias* \\ Centro Multidisciplinar de Astrofísica - CENTRA \\ Departamento de Física, \\ Instituto Superior Técnico - IST, \\ Universidade Técnica de Lisboa - UTL, \\ Av. Rovisco Pais 1, 1049-001 Lisboa, Portugal \\ José P. S. Lemos ${ }^{\dagger}$ \\ Centro Multidisciplinar de Astrofísica - CENTRA \\ Departamento de Física, \\ Instituto Superior Técnico - IST, \\ Universidade Técnica de Lisboa - UTL, \\ Av. Rovisco Pais 1, 1049-001 Lisboa, Portugal
}

\begin{abstract}
The action for a class of three-dimensional dilaton-gravity theories, with an electromagnetic Maxwell field and a cosmological constant, can be recast in a Brans-Dicke-Maxwell type action, with its free $\omega$ parameter. For a negative cosmological constant, these theories have static, electrically charged, and spherically symmetric black hole solutions. Those theories with well formulated asymptotics are studied through a Hamiltonian formalism, and their thermodynamical properties are found out. The theories studied are general relativity $(\omega \rightarrow \pm \infty)$, a dimensionally reduced cylindrical four-dimensional general relativity theory $(\omega=0)$, and a theory representing a class of theories $(\omega=-3)$, all with a Maxwell term. The Hamiltonian formalism is setup in three dimensions through foliations on the right region of the Carter-Penrose diagram, with the bifurcation 1-sphere as the left boundary, and anti-de Sitter infinity as the right boundary. The metric functions on the foliated hypersurfaces and the radial component of the vector potential one-form are the canonical coordinates. The Hamiltonian action is written, the Hamiltonian being a sum of constraints. One finds a new action which yields an unconstrained theory with two pairs of canonical coordinates $\left\{M, P_{M} ; Q, P_{Q}\right\}$, where $M$ is the mass parameter, which for $\omega<-\frac{3}{2}$ and for $\omega= \pm \infty$ needs a careful renormalization, $P_{M}$ is the conjugate momenta of $M, Q$ is the charge parameter, and $P_{Q}$ is its conjugate momentum. The resulting Hamiltonian is a sum of boundary terms only. A quantization of the theory is performed. The Schrödinger evolution operator is constructed, the trace is taken, and the partition function of the grand canonical ensemble is obtained, where the chemical potential is the scalar electric potential $\bar{\phi}$. Like the uncharged cases studied previously, the charged black hole entropies differ, in general, from the usual quarter of the horizon area due to the dilaton.
\end{abstract}

PACS numbers: 04.60.Ds, 04.20.Fy, 04.60.Gw, 04.60.Kz, 04.70.Dy

\section{INTRODUCTION}

In a previous paper [1] we have motivated and studied the Hamiltonian thermodynamics of three-dimensional dilatonic black holes. To construct a classical Hamiltonian formalism is important for several reasons. First, it is always elegant to write the equations of the theory as a pair of symmetric first order equations. Second, if one can put the classical theory into a Hamiltonian form, then by applying certain rules one can get a quantized version of the theory in a first approximation. Third, through Euclideanization of the Schrödinger time evolution operator $\exp (-i H t)$, where $H$ is the Hamiltonian, one gets the partition function, which in turn leads to a thermodynamical decription of the system. Thus, the Hamiltonian formalism and the thermodynamical description are linked subjects. The Hamiltonian thermodynamics of several different black hole systems have been studied first by Louko and Whiting in [2] and further analyzed in [3, 4, [5, 6, 7] and [1] , (see also [8, 9] ). The Louko-Whiting method relies on the Hamiltonian approach of [10], which in turn is an important ramification of the Arnowitt, Deser, and Misner approach, the ADM approach, and its major developments [11, 12]. Other methods, like direct calculation from quantum fields in curved spacetime (see, e.g, [13, 14]), or path integral methods (see, e.g, [15, 16, 17, 18, 19]), have been used to study the thermodynamics of black holes. Now, the study of thermodynamics of black holes in any specific dimension is important, for instance, to understand universal properties independent of the dimension itself. In particular, one can single out three dimensions

\footnotetext{
* Email: gadias@fisica.ist.utl.pt

$\dagger$ Email: lemos@fisica.ist.utl.pt
} 
as an adequate interesting dimension, because three dimensional general relativity has a special black hole with simple properties [20], and dilatonic extensions of this theory have black holes with properties similar to four-dimensional general relativistic black holes [21, 22].

The main purpose of this paper is to generalize our previous work on Hamiltonian thermodynamics of threedimensional dilatonic black holes [1], to black holes, still in three dimensions, in which the graviton-dilaton BransDicke theory, with its $\omega$ parameter, is coupled to a gauge field, namely, the electromagnetic field. Three-dimensional Einstein-Maxwell theory with its black hole solution has been studied in [23] (see also [20]), and electric dilatonic extensions also have charged black holes [24, 25] (see also [21, 22]). Further analyses on the properties of these black holes have been worked out in [26, 27, 28]. In principle, $\omega$ can take any value, i.e., $\infty>\omega>-\infty$. But, since we want to perform a canonical Hamiltonian analysis, using an ADM formalism supplied with proper boundary conditions, it is necessary to pick up only those $\omega_{\text {s }}$ which yield black hole solutions that fulfill boundary conditions adequate to a Hamiltonain method. As in the uncharged case [1], the cases of most interest are then black holes for which $\omega \rightarrow \pm \infty$, $\omega=0$, and $\omega=-3$. Now, unlike the uncharged case, it was realized in [23, 25] (see also [20]) that the inclusion of electric charge creates a complication to the definition of the mass $M$. Indeed, for $\omega<-\frac{3}{2}$, which includes the general relativity case (since it can be considered as the $\omega \rightarrow-\infty$ limit), one finds a divergent asymptotic behavior of the mass. This problem can be cured by a proper mass renormalization [23, 25]. But the whole issue has never been confronted when one makes a full use of the Hamiltonian formalism, as we do here. We solve this difficulty and show that the mass redefinition conforms wholly with the formalism for the $\omega \rightarrow \infty$ case, is not needed in the $\omega=0$ case and it is compatible with the $\omega=-3$ case. In each case, the black hole system and its thermodynamics can then be studied. We have chosen to study the systems in a grand canonical ensemble. In such an ensemble, the boundary radius $l$, the temperature $\mathbf{T}$ (or its inverse $\beta$ ), and the electric potential (i.e., the chemical potential) $\bar{\phi}$ at the boundary radius are fixed. The electric potential is conjugate to the electric charge and, in this ensemble, the charge itself is not fixed. Our main results are, a complete derivation from a Hamiltonian formalism of the mass-energy $\langle E\rangle$, the entropy $S$, and the charge $\langle Q\rangle$, as well as the establishment that the grand canonical ensemble for charged three dimensional black holes is locally stable for the black holes studied. Thus, the choice of such an ensemble is appropriate, since the corresponding boundary onditions lead to a well posed problem and a stable ensemble.

The structure of the paper is as follows. In Sec. II we present the classical solutions of the three-dimensional dilatonic charged black holes, whose quantization through Hamiltonian methods we will perform. There is a free parameter $\omega$ for which we choose three different values, $\omega=\infty, 0,-3$, corresponding to the BTZ black hole, the dimensionally reduce four-dimensional cylindrical black hole, and a three-dimensional dilatonic black hole, respectively. We also introduce the spacetime foliation through which we will define the canonical coordinates and which will allow us to write the action as a sum of constraints multiplied by their respective Lagrange multipliers. Then, follow three sections, Secs. III, IV, and V, where we develop the thermodynamic Hamiltonian formalism for the $\omega=\infty, 0,-3$ charged black holes, respectively. We divide each section into A-F subsections to better analyze the Hamiltonian thermodynamics of the systems.

\section{CHARGED BLACK HOLE SOLUTIONS ALLOWING HAMILTONIAN DESCRIPTION}

\section{A. The 3D black hole solutions}

Three-dimensional theories which contain black holes have been studied in several works. Indeed in [20, 21, 22, 27, 28], a Brans-Dicke action, with gravitational and dilaton fields plus a cosmological constant, was used to study such solutions. Now, we can couple the graviton and dilaton fields to an electromagnetic field. in order to find charged black hole solutions in three dimensions. For three-dimensional general relativity this has been done in [23] (see also [20]), whereas for Brans-Dicke theory this has been done in [25] (see also [28]). The Brans-Dicke action with a Maxwell term is given by

$$
S=\frac{1}{2 \pi} \int d^{3} x \sqrt{-g} e^{-2 \phi}\left(R-4 \omega(\partial \phi)^{2}+4 \lambda^{2}+F^{\mu \nu} F_{\mu \nu}\right)+\bar{B}
$$

where $g$ is the determinant of the three-dimensional metric $g_{\mu \nu}, R$ is the curvature scalar, $\phi$ is a scalar dilaton field, $\omega$ is the Brans-Dicke parameter, $\lambda$ is the cosmological constant, $F_{\mu \nu}=\partial_{\mu} A_{\nu}-\partial_{\nu} A_{\mu}$ is the Maxwell tensor, where $A=A_{\mu} d x^{\mu}$ is the vector potential one-form, and $\bar{B}$ is a generic surface term. A general solution is a solution for the metric, the dilaton and the gauge field. We search for static solutions. The solution for the metric is then [25]

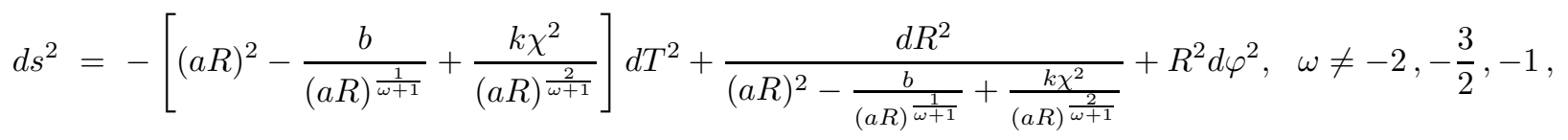




$$
\begin{array}{ll}
d s^{2}=-\left[\left(1+\frac{\chi^{2}}{4} \ln R\right) R^{2}-b R\right] d T^{2}+\frac{d R^{2}}{\left(1+\frac{\chi^{2}}{4} \ln R\right) R^{2}-b R}+R^{2} d \varphi^{2} & \omega=-2, \\
d s^{2}=\left(4 \lambda^{2} R^{2} \ln (b R)+\chi^{2} R^{4}\right) d T^{2}-\frac{d R^{2}}{4 \lambda^{2} R^{2} \ln (b R)+\chi^{2} R^{4}}+R^{2} d \varphi^{2}, & \omega=-\frac{3}{2}, \\
d s^{2}=-d T^{2}+d R^{2}+d \varphi^{2}, & \omega=-1,
\end{array}
$$

where $T, R$ are Schwarzschild coordinates, $a$ is a constant related to the cosmological constant (see below), and $b$ is a constant of integration related to the mass (see below), $\chi$ is constant of integration related to the electric charge, and $k$ is defined as $k=\frac{1}{8} \frac{(\omega+1)^{2}}{(\omega+2)}$. The general solution for $\phi$ is given by

$$
\begin{array}{ll}
\phi=-\frac{1}{2(\omega+1)} \ln (a R), & \omega \neq-1, \\
\phi=\text { constant }, & \omega=-1 .
\end{array}
$$

The general solution for the vector potential $A$, written as $A=A_{\mu}(r) d x^{\mu}=A_{T}(r) d T$, is given by

$$
\begin{array}{ll}
A & =\frac{1}{4} \chi(\omega+1)(a R)^{-\frac{1}{\omega+1}} d T, \quad \omega \neq-1, \\
A & =0, \quad \omega=-1 .
\end{array}
$$

For the constant $a$ one has

$$
\begin{array}{ll}
a=\frac{2|(\omega+1) \lambda|}{\sqrt{|(\omega+2)(2 \omega+3)|},} & \omega \neq-2,-\frac{3}{2},-1, \\
a=1, & \omega=-2,-\frac{3}{2}, \\
a=0, & \omega=-1 .
\end{array}
$$

For $\omega=-2$ we have $\lambda^{2}=-\chi^{2} / 16$, which means that, unlike for the uncharged case, the cosmological constant is not null. The constant $b$ is related to the ADM mass of the solutions by

$$
\begin{array}{ll}
M=\frac{\omega+2}{\omega+1} b, & \omega \neq-2,-\frac{3}{2},-1, \\
M=-b & \omega=-2, \\
M=-4 \lambda^{2} \ln b, & \omega=-\frac{3}{2}, \\
M=0, & \omega=-1 .
\end{array}
$$

The constant $\chi$ is s related to the ADM charge of the solutions by

$$
\begin{array}{ll}
\chi^{2}=Q^{2}, & \omega \neq-2,-\frac{3}{2},-1, \\
\chi^{2}=0, & \omega=-1 .
\end{array}
$$

For $\omega=-2,-\frac{3}{2}$, there is no good definition for $Q$ in terms of $\chi$.

Since we want to perform a canonical Hamiltonian analysis, using an ADM formalism supplied with proper boundary conditions, it is necessary to pick up only those solutions that fulfill the conditions we want to impose. First, we are interested only in solutions with horizons, so we take $b$ to be positive. Second, apart from a measure zero of solutions, all solutions have a non-zero $|\lambda|$. This does not mean straight away that the solutions are asymptotically anti-de Sitter. Some have one type or another of singularities at infinity, which do not allow an imposition of proper boundary conditions. So, from [25] with the corresponding Carter-Penrose diagrams we discard the following solutions: $\omega=-1$ which is simply the Minkowski solution, $-1>\omega>-\frac{3}{2}$ since it has a curvature singularity at $R=+\infty$, which is inside the horizon, and has a null topological singularity at $R=0, \omega=-\frac{3}{2}$ since all the Carter-Penrose boundary is singular, the same happening in the interval $-\frac{3}{2}>\omega>-2$. Thus, the cases of interest to be studied are black holes for which $\infty>\omega>-1$ and $-2>\omega>-\infty$. For $b$ positive these solutions have ADM mass $M$ positive. As in [1] we choose three typical amenable cases where an analytical study can be done. The first case is the charged BTZ black hole [23] (see also [20]), which can be found by taking appropriately the limit $\omega \rightarrow \pm \infty$. This black hole is thus a 
solution of the Einstein-Maxwell action in three dimensions. The other cases are $\omega=0$ and $\omega=-3$. The theory with $\omega=0$ is equivalent to cylindrical four-dimensional Einstein-Maxwell general relativity, and the corresponding charge black hole was found in [24], see also [25]. The theory with $\omega=-3$ is just a case of $3 \mathrm{D}$ charged Brans-Dicke theory, with a black hole solution that can be analyzed in this context [25].

Now, as mentioned in the introduction, the definition of the ADM mass $M$ is not straightforward when there is electric charge, in the sense that one can face a divergent asymptotic behavior of the mass $M$, see [23, 25] as well as [20]. The problem is more acute when $\omega<-\frac{3}{2}$, which includes the general relativity case (since general relativity can be considered the $\omega \rightarrow-\infty$ ), see [25] for a full discussion (see also [20, 23]). For such $\omega$, the mass $M$ can be written as $M=M_{Q=0}+\operatorname{Div}_{M}(\chi, R)$, where $M_{Q=0}$ is the mass for the uncharged case and Div $M(\chi, R)$ is the divergent part when there is charge. To treat this divergence, as explained in [25], we consider a large radius $R_{*}$ frontier. Then we write the mass $M$ as

$$
M=M\left(R_{*}\right)+\left[\operatorname{Div}_{M}(\chi, R)-\operatorname{Div}_{M}\left(\chi, R_{*}\right)\right],
$$

where the function $M\left(R_{*}\right)$ is equal to

$$
M\left(R_{*}\right)=M_{Q=0}+\operatorname{Div}_{M}\left(\chi, R_{*}\right) .
$$

The term in square brackets in Eq. (19) tends to zero as the radius $R$ tends to the frontier radius, $R \rightarrow R_{*} . M\left(R_{*}\right)$ is interpreted as the energy inside the radius $R_{*}$. From Eq. (20) the term $-\operatorname{Div}_{M}\left(\chi, R_{*}\right)$ is interpreted as the energy outside the frontier in $R_{*}$, apart from an infinite constant, which is hidden in $M\left(R_{*}\right)$. The total mass definition, $M$, is then well defined. In practical terms, one needs not pay attention to the divergent factors Div $M$ because all one needs to do is consider null the asymptotic $\operatorname{limits}, \lim \operatorname{Div}_{M}(\chi, R)=0$, ignoring the frontier at $R_{*}$. For $\omega>-3 / 2$ the mass term behavior of the three-dimensional black holes is similar to the Reissner-Nordström case, and it offers no problems. Indeed, for the Reissner-Nordström black hole the metric function $g_{t t}$ and the mass term, following the prescription above, are written as $g_{t t}=1-\frac{2 M\left(R_{*}\right)}{R}+Q^{2}\left(\frac{1}{R}-\frac{1}{R_{*}}\right)$, and $M=M\left(R_{*}\right)+\frac{Q^{2}}{2 R_{*}}$, respectively. The energy inside the frontier $R_{*}$ is then given by $M\left(R_{*}\right)$, and the electrostatic energy outside is given by $\frac{Q^{2}}{2 R_{*}}$. As this last term tends to zero as $R_{*} \rightarrow \infty$ there are no real divergences in this case, thus implying that $M\left(R_{*}\right)$ carries no infinity within, and so the prescription is trivial, it gves nothing new. This is similar to what happens for three-dimensional black holes with $\omega>-3 / 2$. On the other hand, for $\omega<-3 / 2$ one should follow the prescription carefully, i.e., one has to hide an infinite constant in the definition of the energy within the frontier at $R_{*}$.

\section{B. ADM form of the metric and dilaton}

The ansatz for the metric and dilaton fields with which we start our canonical analysis is given by

$$
\begin{gathered}
d s^{2}=-N(t, r)^{2} d t^{2}+\Lambda(t, r)^{2}\left(d r+N^{r}(t, r) d t\right)^{2}+R(t, r)^{2} d \varphi^{2} \\
\mathrm{e}^{-2 \phi}=(a R(t, r))^{\frac{1}{\omega+1}} .
\end{gathered}
$$

where $\{T, R, \varphi\}$ are the Schwarzschild type time, radius, and angular coordinates. This is the ADM ansatz for spherically symmetric solutions applied to three dimensions. In this we follow the basic formalism developed by Kuchař [10]. The canonical coordinates $R$ and $\Lambda$ are functions of $t$ and $r$, i.e., $R=R(t, r)$ and $\Lambda=\Lambda(t, r)$. Now, $r=0$ is generically on the horizon as analyzed in [10], but for our purposes $r=0$ represents the horizon bifurcation point of the Carter-Penrose diagram [2] (see also [3]-[6] and [1]). In three spacetime dimensions the point actually represents a circle. Asymptotically the coordinate $r$ tends to $\infty$, and $t$ is another time coordinate. The remaining functions are the lapse function $N=N(t, r)$, and the shift function $N^{r}=N^{r}(t, r)$, and they will play the role of Lagrange multipliers of the Hamiltonian of the theory. The canonical coordinates $R=R(t, r), \Lambda=\Lambda(t, r)$ and the lapse function $N=N(t, r)$ are taken to be positive. The angular coordinates are left untouched, due to spherical symmetry. The dilaton is a simple function of the radial canonical coordinate, and it can be traded directly by it through equation (22), as will be done below. The ansatz (21)-(22) is written in order to perform the foliation of spacetime into spacelike hypersurfaces, and thus separates the spatial part of the spacetime from the temporal part. Indeed, the canonical analysis requires the explicit separation of the time coordinate from the other space coordinates, and so in all expressions time is treated separately from the other coordinates. It breaks explicit, but not implicit, covariance of the theory, but it is necessary in order to perform the Hamiltonian analysis. The metric coefficients of the induced metric on the hypersurfaces become the canonical variables, and the momenta are determined in the 
usual way, by replacing the time derivatives of the canonical variables, the velocities. Then, using the Hamiltonian, one builds a time evolution operator to construct an appropriate thermodynamic ensemble for the geometries of a quantum theory of gravity. Assuming that a quantum theory only makes sense if its classical form can be quantized by Hamiltonian methods, one should pick up only solutions which can be put consistently in a Hamiltonian form. Thus, in the following we perform a Hamiltonian analysis to extract the entropy and other thermodynamic properties in the three-dimensional Brans-Dicke-Maxwell black holes mentioned above, those for which $\omega=\infty, 0,-3$.

\section{ADM form for the vector potential $A$}

The canonical description of the vector potential one-form is given by

$$
A=\Gamma d r+\Phi d t
$$

where $\Gamma$ and $\Phi$ are functions of $t$ and $r$, i. e., $\Gamma(t, r)$ and $\Phi(t, r)$. The function $\Gamma(t, r)$ is the canonical coordinate associated with the electric field, and the function $\Phi(t, r)$ is the Lagrange multiplier associated with the electromagnetic constraint, which is Gauss' Law. In the same way as with the gravitational degrees of freedom above, when the Maxwell term in the action (1) is written, the conjugate momentum to the coordinate $\Gamma(t, r)$ can be derived the usual way from the Lagrangian.

\section{HAMILTONIAN THERMODYNAMICS OF THE CHARGED BTZ BLACK HOLE $(\omega=\infty)$}

\section{A. The metric and the vector potential one-form}

For $\omega=\infty$, the corresponding three-dimensional charged Brans-Dicke theory is precisely the one provided by threedimensional Einstein-Maxwell theory [20, 23, 25]. Then the general metric in Eq. (2), the $\phi$ field in Eq. (6), and the vector potential one-form (8) reduce to the following

$$
\begin{aligned}
d s^{2} & =-\left(\frac{R^{2}}{l^{2}}-M-\pi Q^{2} \ln \left(\frac{R}{l}\right)\right) d T^{2}+\frac{d R^{2}}{\frac{R^{2}}{l^{2}}-M-\pi Q^{2} \ln \left(\frac{R}{l}\right)}+R^{2} d \varphi^{2} \\
\mathrm{e}^{-2 \phi} & =1 \\
A & =\frac{1}{2 \pi} Q \ln \left(\frac{R}{l}\right) d T
\end{aligned}
$$

where $l$ is the AdS length, related to the cosmological constant by $2 \lambda^{2}=l^{-2}, M$ is the mass, and $Q$ is the electric charge. In this solution the scalar field $\phi$ is trivial. Next, in Fig. 1, we show the Carter-Penrose diagram of the three-dimensional charged black hole. Despite there being a large and rich structure, the outside region in $\mathrm{I}$ is the relevant one, thus our foliation is from the bifurcation point to $R=\infty$, and our boundary conditions reflect properties of the outer horizon at $R=R_{\mathrm{h}}$, and of the spacelike infinity at $R=\infty$.

\section{B. Canonical formalism}

Given Eqs. (21)-(23), the three-dimensional action (1), now an Einstein-Maxwell action, becomes, excluding surface terms,

$$
\begin{aligned}
S\left[\Lambda, R, \Gamma, \dot{\Lambda}, \dot{R}, \dot{\Gamma} ; N, N^{r}, \Phi\right]= & \int d t \int_{0}^{\infty} d r\left\{-2 N^{-1} \dot{\Lambda} \dot{R}+2 N^{-1} N^{r} R^{\prime} \dot{\Lambda}+2 N^{-1}\left(N^{r}\right)^{\prime} \Lambda \dot{R}+2 N^{-1} N^{r} \Lambda^{\prime} \dot{R}\right. \\
& -2 N^{-1}\left(N^{r}\right)^{2} \Lambda^{\prime} R^{\prime}+2 N \Lambda^{-2} \Lambda^{\prime} R^{\prime}-2 N \Lambda^{-1} R^{\prime \prime}+4 N \lambda^{2} \Lambda R \\
& \left.+\pi N^{-1} \Lambda^{-1} R\left(\dot{\Gamma}-\Phi^{\prime}\right)^{2}\right\}
\end{aligned}
$$

where $2 \lambda^{2}=l^{-2}$ and $l$ is the AdS length, which then yields the quantity $a$ is $a^{2}=2 \lambda^{2}=l^{-2}$. In Eq. (27) a means derivative with respect to time $t$ and $\mathrm{a}^{\prime}$ means derivative with respect to $r$, and where all the explicit functional 


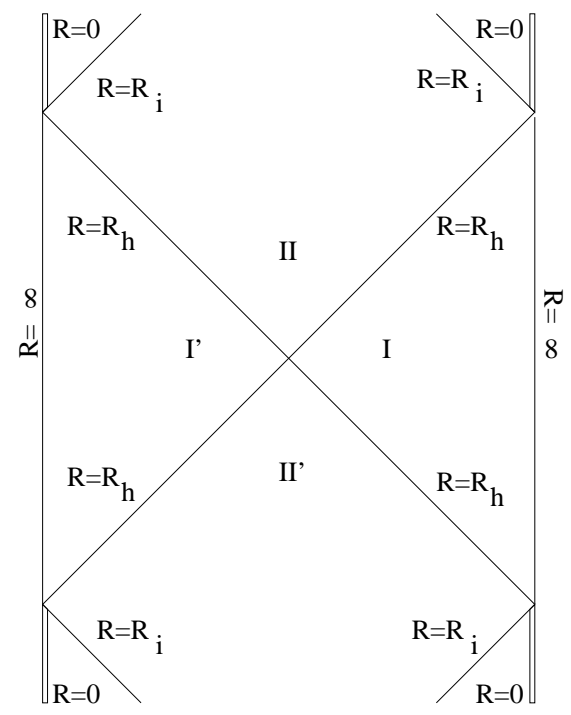

FIG. 1: The Carter-Penrose diagram for the charged BTZ case, where $R_{\mathrm{h}}$ is the outer horizon radius, $R_{\mathrm{i}}$ is the inner horizon radius, and the double line is the timelike singularity at $R=0$. The static region $\mathrm{I}$, from the bifurcation point to spacelike infinity $R=\infty$, is the relevant region for our analysis. The regions II and II' are inside the outer horizon, beyond the foliation domain. Region I' is the symmetric of region I, towards left infinity.

dependences are omitted. From the action (27) one determines the canonical momenta, conjugate to $\Lambda, R$, and $\Gamma$ respectively,

$$
\begin{aligned}
& P_{\Lambda}=-2 N^{-1}\left\{\dot{R}-R^{\prime} N^{r}\right\}, \\
& P_{R}=-2 N^{-1}\left\{\dot{\Lambda}-\left(\Lambda N^{r}\right)^{\prime}\right\}, \\
& P_{\Gamma}=2 \pi N^{-1} \Lambda^{-1} R\left(\dot{\Gamma}-\Phi^{\prime}\right) .
\end{aligned}
$$

By performing a Legendre transformation, we obtain

$$
\begin{aligned}
\mathcal{H}= & N\left\{-\frac{1}{2} P_{R} P_{\Lambda}+\frac{1}{4 \pi} P_{\Gamma}^{2} \Lambda R^{-1}-2 \Lambda^{-2} \Lambda^{\prime} R^{\prime}+2 \Lambda^{-1} R^{\prime \prime}-4 \lambda^{2} \Lambda R\right\}+N^{r}\left\{P_{R} R^{\prime}-P_{\Lambda}^{\prime} \Lambda-\Gamma P_{\Gamma}^{\prime}\right\}+\tilde{\Phi}\left\{-P_{\Gamma}^{\prime}\right\} \\
& \equiv N H+N^{r} H_{r}+\tilde{\Phi} G,
\end{aligned}
$$

defining implicitly in this way the constraints $H$ and $H_{r}$, and $G$, and where the new Lagrange multiplier is $\tilde{\Phi} \equiv \Phi-N^{r} \Gamma$. Here additional surface terms have been ignored, as for now we are interested in the bulk terms only. The action in Hamiltonian form is then

$$
S\left[\Lambda, R, \Gamma, P_{\Lambda}, P_{R}, P_{\Gamma} ; N, N^{r}, \tilde{\Phi}\right]=\int d t \int_{0}^{\infty} d r\left\{P_{\Lambda} \dot{\Lambda}+P_{R} \dot{R}+P_{\Gamma} \dot{\Gamma}-N H-N^{r} H_{r}-\tilde{\Phi} G\right\}
$$

The equations of motion are

$$
\begin{aligned}
\dot{\Lambda} & =-\frac{1}{2} N P_{R}+\left(N^{r} \Lambda\right)^{\prime} \\
\dot{R} & =-\frac{1}{2} N P_{\Lambda}-N^{r} R^{\prime} \\
\dot{P_{R}} & =4 \lambda^{2} N \Lambda-\left(2 N^{\prime} \Lambda^{-1}\right)^{\prime}+\left(N^{r} P_{R}\right)^{\prime}+\frac{1}{4 \pi} P_{\Gamma}^{2} N \Lambda R^{-2}, \\
\dot{P_{\Lambda}} & =4 \lambda^{2} N R-2 N^{\prime} \Lambda^{-2} R^{\prime}+N^{r}\left(P_{\Lambda}\right)^{\prime}-\frac{1}{4 \pi} P_{\Gamma}^{2} N R^{-1}, \\
\dot{\Gamma} & =\frac{1}{2 \pi} P_{\Gamma} N \Lambda R^{-1}+\left(N^{r} \Gamma\right)^{\prime}+\tilde{\Phi}^{\prime}, \\
\dot{P_{\Gamma}} & =N^{r} P_{\Gamma}^{\prime} .
\end{aligned}
$$


In order to have a well defined variational principle, we need to eliminate the surface terms of the original bulk action, which render the original action itself ill defined when one seeks a correct determination of the equations of motion through variational methods. These surface terms are eliminated through judicious choice of extra surface terms which should be added to the action. The action (32) has the following extra surface terms, after variation

$$
\text { Surface terms }=\left.\left(-N^{r} P_{R} \delta R+N^{r} \Lambda \delta P_{\Lambda}+2 N \Lambda^{-2} R^{\prime} \delta \Lambda-2 N \Lambda^{-1} \delta R^{\prime}+2 N^{\prime} \Lambda^{-1} \delta R+N^{r} \Gamma \delta P_{\Gamma}+\tilde{\Phi} \delta P_{\Gamma}\right)\right|_{0} ^{\infty} .
$$

In order to evaluate this expression, we need to know the asymptotic conditions of each of the above functions individually, which are functions of $(t, r)$.

Starting with the limit $r \rightarrow 0$ we assume

$$
\begin{aligned}
\Lambda(t, r) & =\Lambda_{0}+O\left(r^{2}\right), \\
R(t, r) & =R_{0}+R_{2} r^{2}+O\left(r^{4}\right), \\
P_{\Lambda}(t, r) & =O\left(r^{3}\right), \\
P_{R}(t, r) & =O(r), \\
N(t, r) & =N_{1}(t) r+O\left(r^{3}\right), \\
N^{r}(t, r) & =O\left(r^{3}\right), \\
\Gamma(t, r) & =O(r), \\
P_{\Gamma}(t, r) & =2 \pi Q_{0}+2 \pi Q_{2} r^{2}+O\left(r^{4}\right), \\
\tilde{\Phi}(t, r) & =\tilde{\Phi}_{0}(t)+O\left(r^{2}\right) .
\end{aligned}
$$

Note that there are time dependences on the left hand side of the falloff conditions and that there are no such dependences on the right hand side, in the lower orders of the expansion in $r$. This apparent discrepancy stems from the fact that there is in fact no time dependence in the lower orders of the majority of the functions, but there may still exist such dependence for higher orders. Nevertheless, terms such as $R_{0}$ are functions, independent of $(t, r)$, thus constant, but undetermined. Their variation makes sense, as we may still vary between different values for these constant functions. With these conditions, we have for the surface terms at $r=0$

$$
\text { Surface terms }\left.\right|_{r=0}=-2 N_{1} \Lambda_{0}^{-1} \delta R_{0}-\tilde{\Phi}_{0} \delta\left(2 \pi Q_{0}\right) \text {. }
$$

For $r \rightarrow \infty$ we have

$$
\begin{aligned}
\Lambda(t, r) & =l r^{-1}+l^{3} \eta(t) r^{-3}+O^{\infty}\left(r^{-5}\right), \\
R(t, r) & =r+l^{2} \rho(t) r^{-1}+O^{\infty}\left(r^{-3}\right), \\
P_{\Lambda}(t, r) & =O^{\infty}\left(r^{-2}\right), \\
P_{R}(t, r) & =O^{\infty}\left(r^{-4}\right), \\
N(t, r) & =R(t, r)^{\prime} \Lambda(t, r)^{-1}\left(\tilde{N}_{+}(t)+O^{\infty}\left(r^{-5}\right)\right), \\
N^{r}(t, r) & =O^{\infty}\left(r^{-2}\right), \\
\Gamma(t, r) & =O^{\infty}\left(r^{-2}\right), \\
P_{\Gamma}(t, r) & =2 \pi Q_{+}(t)+O^{\infty}\left(r^{-2}\right), \\
\tilde{\Phi}(t, r) & =\tilde{\Phi}_{+}(t)+O^{\infty}\left(r^{-1}\right) .
\end{aligned}
$$

These conditions imply for the surface terms in the limit $r \rightarrow \infty$

$$
\text { Surface terms }\left.\right|_{r \rightarrow \infty}=2 \delta\left(M_{+}(t)\right) \tilde{N}_{+}+\tilde{\Phi}_{+} \delta\left(2 \pi Q_{+}\right),
$$

where $M_{+}(t)=2(\eta(t)+2 \rho(t))$. So, the surface term added to (32) is

$$
S_{\partial \Sigma}\left[\Lambda, R, Q_{0}, Q_{+} ; N, \tilde{\Phi}_{0}, \tilde{\Phi}_{+}\right]=\int d t\left(2 R_{0} N_{1} \Lambda_{0}^{-1}-\tilde{N}_{+} M_{+}+\tilde{\Phi}_{0}\left(2 \pi Q_{0}\right)-\tilde{\Phi}_{+}\left(2 \pi Q_{+}\right)\right) .
$$

What is left after varying this last surface term and adding it to the varied initial action (see Eq. (32) is

$$
\int d t\left(2 R_{0} \delta\left(N_{1} \Lambda_{0}^{-1}\right)-M_{+} \delta \tilde{N}_{+}+2 \pi Q_{0} \delta \tilde{\Phi}_{0}-2 \pi Q_{+} \delta \tilde{\Phi}_{+}\right) .
$$


We choose to fix $N_{1} \Lambda_{0}^{-1}$ and $\tilde{\Phi}_{0}$ on the horizon $(r=0)$, and $\tilde{N}_{+}$and $\tilde{\Phi}_{0}$ at infinity. These choices make the surface variation (61) disappear. The term $N_{1} \Lambda_{0}^{-1}$ is the integrand of

$$
n^{a}\left(t_{1}\right) n_{a}\left(t_{2}\right)=-\cosh \left(\int_{t_{1}}^{t_{2}} d t N_{1}(t) \Lambda_{0}^{-1}(t)\right),
$$

which is the rate of the boost suffered by the future unit normal to the constant $t$ hypersurfaces defined at the bifurcation circle, i.e., at $r \rightarrow 0$, due to the evolution of the constant $t$ hypersurfaces. By fixing the integrand we are fixing the rate of the boost, which allows us to control the metric singularity when $r \rightarrow 0$ [2]. Finally, we also fix $\tilde{\Phi}_{0}$ and $\tilde{\Phi}_{+}$at $r=0$ and at infinity, respectively.

\section{Reconstruction, canonical transformation, and action}

In order to reconstruct the mass and the time from the canonical data, which amounts to making a canonical transformation, we have to rewrite the form of the solutions of Eqs. (24)-(26). However, in addition to reconstructing the mass as done in [1], we have to consider how to reconstruct the charge from the canonical data. We follow Kuchař [10] for this reconstruction. We concentrate our analysis on the right static region of the Carter-Penrose diagram.

Developing the Killing time $T$ as function of $(t, r)$ in the expression for the vector potential (26), and making use of the gauge freedom that allows us to write

$$
A=\frac{1}{2 \pi} Q \ln \left(\frac{R}{l}\right) d T+d \xi
$$

where $\xi(t, r)$ is an arbitrary continuous function of $t$ and $r$, we can write the one-form potential as

$$
A=\left(\frac{1}{2 \pi} Q \ln \left(\frac{R}{l}\right) T^{\prime}+\xi^{\prime}\right) d r+\left(\frac{1}{2 \pi} Q \ln \left(\frac{R}{l}\right) \dot{T}+\dot{\xi}\right) d t .
$$

Equating the expression (64) with Eq. (23), and making use of the definition of $P_{\Gamma}$ in Eq. (30), we arrive at

$$
P_{\Gamma}=2 \pi Q .
$$

In the static region, we define $F$ as

$$
F(t, r)=\frac{R^{2}}{l^{2}}-M-\frac{1}{4 \pi} P_{\Gamma}^{2} \ln \left(\frac{R}{R_{*}}\right),
$$

where we define the $R_{*}$ as the (large) radius of a finite frontier, which will serve as a renormalization of the asymptotic properties of the future canonical coordinate $M(t, r)$ (see subsection IA ). This frontier will be used throughout the present section. We now make the following substitutions

$$
T=T(t, r), \quad R=R(t, r),
$$

into the solution (24), getting

$$
d s^{2}=-\left(F \dot{T}^{2}-F^{-1} \dot{R}^{2}\right) d t^{2}+2\left(-F T^{\prime} \dot{T}+F^{-1} R^{\prime} \dot{R}\right) d t d r+\left(-F\left(T^{\prime}\right)^{2}+F^{-1} \dot{R}^{2}\right) d r^{2}+R^{2} d \varphi^{2} .
$$

This introduces the ADM foliation directly into the solutions. Comparing it with the ADM metric (21), written in another form as

$$
d s^{2}=-\left(N^{2}-\Lambda^{2}\left(N^{r}\right)^{2}\right) d t^{2}+2 \Lambda^{2} N^{r} d t d r+\Lambda^{2} d r^{2}+R^{2} d \varphi^{2},
$$

we can write a set of three equations

$$
\begin{aligned}
\Lambda^{2} & =-F\left(T^{\prime}\right)^{2}+F^{-1}\left(R^{\prime}\right)^{2}, \\
\Lambda^{2} N^{r} & =-F T^{\prime} \dot{T}+F^{-1} R^{\prime} \dot{R}, \\
N^{2}-\Lambda^{2}\left(N^{r}\right)^{2} & =F \dot{T}^{2}-F^{-1} \dot{R}^{2} .
\end{aligned}
$$


The first two equations, Eqs. (70) and Eq. (171), give

$$
N^{r}=\frac{-F T^{\prime} \dot{T}+F^{-1} R^{\prime} \dot{R}}{-F\left(T^{\prime}\right)^{2}+F^{-1}\left(R^{\prime}\right)^{2}} .
$$

This one solution, together with Eq. (70), give

$$
N=\frac{R^{\prime} \dot{T}-T^{\prime} \dot{R}}{\sqrt{-F\left(T^{\prime}\right)^{2}+F^{-1}\left(R^{\prime}\right)^{2}}} .
$$

One can show that $N(t, r)$ is positive (see [10]). Next, putting Eqs. (73)-(74), into the definition of the conjugate momentum of the canonical coordinate $\Lambda$, given in Eq. (28), one finds the spatial derivative of $T(t, r)$ as a function of the canonical coordinates, i.e.,

$$
-T^{\prime}=\frac{1}{2} F^{-1} \Lambda P_{\Lambda} .
$$

Later we will see that $-T^{\prime}=P_{M}$, as it will be conjugate to a new canonical coordinate $M$. Following this procedure to the end, we may then find the form of the new coordinate $M(t, r)$, as a function of $t$ and $r$. First, we need to know the form of $F$ as a function of the canonical pair $\Lambda, R$. For that, we replace back into Eq. (70) the definition, in Eq. (75), of $T^{\prime}$, giving

$$
F=\left(\frac{R^{\prime}}{\Lambda}\right)^{2}-\left(\frac{P_{\Lambda}}{2}\right)^{2}
$$

Equating this form of $F$ with Eq. (66), we obtain

$$
M=\frac{R^{2}}{l^{2}}-\frac{1}{4 \pi} P_{\Gamma}^{2} \ln \left(\frac{R}{R_{*}}\right)-F,
$$

where $F$ is given in Eq. (76). We thus have found the form of the new canonical coordinate, $M$. It is now a straightforward calculation to determine the Poisson bracket of this variable with $P_{M}=-T^{\prime}$ and see that they are conjugate, thus making Eq. (75) the conjugate momentum of $M$, i.e.,

$$
P_{M}=\frac{1}{2} F^{-1} \Lambda P_{\Lambda}
$$

The other natural transformation has been given in (65), namely the one that relates $P_{\Gamma}$ with $Q$ through $P_{\Gamma}=2 \pi Q$. As $M, Q$ is also a natural choice for canonical coordinate, being another physical parameter of the solution (24). The one coordinate which remains to be found is $P_{Q}$, the conjugate momentum to the charge. It is also necessary to find out the other new canonical variable which commutes with $M, P_{M}$, and $Q$, and which guarantees, with its conjugate momentum, that the transformation from $\Lambda, R, \Gamma$, to $M, Q$, and the new variable is canonical. Immediately it is seen that $R$ commutes with $M, P_{M}$, and $Q$. It is then a candidate. It remains to be seen whether $P_{R}$ also commutes with $M, P_{M}$, and $Q$. As with $R$, it is straightforward to see that $P_{R}$ does not commute with $M$ and $P_{M}$, as these contain powers of $R$ in their definitions, and $\left\{R(t, r), P_{R}\left(t, r^{*}\right)\right\}=\delta\left(r-r^{*}\right)$. So, rename the canonical variable $R$ as $R=\mathrm{R}$. We have then to find a new conjugate momentum to $\mathrm{R}$ which also commutes with $M, P_{M}$, and $Q$, making the transformation from $\left\{\Lambda, R, \Gamma ; P_{\Lambda}, P_{R}, P_{\Gamma}\right\} \rightarrow\left\{M, \mathrm{R}, Q ; P_{M}, P_{\mathrm{R}}, P_{Q}\right\}$ a canonical one. The way to proceed is to look at the constraint $H_{r}$, which is called in this formalism the super-momentum. This is the constraint which generates spatial diffeomorphisms in all variables. Its form, in the initial canonical coordinates, is $H_{r}=-\Lambda P_{\Lambda}^{\prime}+P_{R} R^{\prime}-\Gamma P_{\Gamma}^{\prime}$. In this formulation, $\Lambda$ is a spatial density, $R$ is a spatial scalar, and $\Gamma$ is also a spatial density. As the new variables, $M, \mathrm{R}$, and $Q$, are spatial scalars, the generator of spatial diffeomorphisms is written as $H_{r}=P_{M} M^{\prime}+P_{\mathrm{R}} \mathrm{R}^{\prime}+P_{Q} Q^{\prime}$, regardless of the particular form of the canonical coordinate transformation. It is thus equating these two expressions of the super-momentum $H_{r}$, with $M, P_{M}$, and $Q$ written as functions of $\Lambda, R, \Gamma$ and their respective momenta, that gives us one equation for the new $P_{\mathrm{R}}$ and $P_{Q}$. This means that we have two unknowns, $P_{\mathrm{R}}$ and $P_{Q}$, for one equation only. This sugests that we should make the coefficients of $R^{\prime}$ and $P_{\Gamma}^{\prime}$ equal to zero independently. This results in

$$
\begin{aligned}
P_{\mathrm{R}}= & P_{R}-2 \lambda^{2} F^{-1} \Lambda P_{\Lambda} R+F^{-1} \Lambda^{-1} P_{\Lambda} R^{\prime \prime}-F^{-1} \Lambda^{-2} P_{\Lambda} \Lambda^{\prime} R^{\prime} \\
& -F^{-1} \Lambda^{-1} P_{\Lambda}^{\prime} R^{\prime}+\frac{1}{8 \pi} P_{\Gamma}^{2} R^{-1} F^{-1} \Lambda P_{\Lambda}, \\
P_{Q}= & -2 \pi \Gamma+\frac{1}{2} P_{\Gamma} \ln \left(\frac{R}{R_{*}}\right) F^{-1} \Lambda P_{\Lambda} .
\end{aligned}
$$


It can be shown that $P_{Q}$ commutes with the new $P_{\mathrm{R}}$ and with the rest of the new coordinates, except with $Q$. We have now all the canonical variables of the new set determined. For completeness and future use, we write the inverse transformation for $\Lambda$ and $P_{\Lambda}$,

$$
\begin{aligned}
\Lambda & =\left(\left(\mathrm{R}^{\prime}\right)^{2} F^{-1}-P_{M}^{2} F\right)^{\frac{1}{2}}, \\
P_{\Lambda} & =2 F P_{M}\left(\left(\mathrm{R}^{\prime}\right)^{2} F^{-1}-P_{M}^{2} F\right)^{-\frac{1}{2}} .
\end{aligned}
$$

In summary, the canonical transformations are the following,

$$
\begin{aligned}
\mathrm{R}= & R \\
M= & \frac{R^{2}}{l^{2}}-\frac{1}{4 \pi} P_{\Gamma}^{2} \ln \left(\frac{R}{R_{*}}\right)-F \\
Q= & \frac{P_{\Gamma}}{2 \pi} \\
P_{\mathrm{R}}= & P_{R}-2 \lambda^{2} F^{-1} \Lambda P_{\Lambda} R+F^{-1} \Lambda^{-1} P_{\Lambda} R^{\prime \prime}-F^{-1} \Lambda^{-2} P_{\Lambda} \Lambda^{\prime} R^{\prime} \\
& -F^{-1} \Lambda^{-1} P_{\Lambda}^{\prime} R^{\prime}+\frac{1}{8 \pi} P_{\Gamma}^{2} R^{-1} F^{-1} \Lambda P_{\Lambda}, \\
P_{M}= & \frac{1}{2} F^{-1} \Lambda P_{\Lambda}, \\
P_{Q}= & -2 \pi \Gamma+\frac{1}{2} P_{\Gamma} \ln \left(\frac{R}{R_{*}}\right) F^{-1} \Lambda P_{\Lambda} .
\end{aligned}
$$

It remains to be seen that this set of transformations is in fact canonical. In order to prove that the set of equalities in expression (83) is canonical we start with the equality

$$
\begin{aligned}
P_{\Lambda} \delta \Lambda+P_{R} \delta R+P_{\Gamma} \delta \Gamma-P_{M} \delta M-P_{\mathrm{R}} \delta \mathrm{R}-P_{Q} \delta Q= & \left(\delta R \ln \left|\frac{2 R^{\prime}+\Lambda P_{\Lambda}}{2 R^{\prime}-\Lambda P_{\Lambda}}\right|\right)^{\prime}+ \\
& +\delta\left(\Gamma P_{\Gamma}+\Lambda P_{\Lambda}+R^{\prime} \ln \left|\frac{2 R^{\prime}-\Lambda P_{\Lambda}}{2 R^{\prime}+\Lambda P_{\Lambda}}\right|\right)
\end{aligned}
$$

We now integrate expression (84) in $r$, in the interval from $r=0$ to $r=\infty$. The first term on the right hand side of Eq. (84) vanishes due to the falloff conditions (see Eqs. (40)-(48) and Eqs. (50)-(58)). We then obtain the following expression

$$
\int_{0}^{\infty} d r\left(P_{\Lambda} \delta \Lambda+P_{R} \delta R+P_{\Gamma} \delta \Gamma\right)-\int_{0}^{\infty} d r\left(P_{M} \delta M+P_{\mathrm{R}} \delta \mathrm{R}+P_{Q} \delta Q\right)=\delta \omega\left[\Lambda, R, \Gamma, P_{\Lambda}, P_{\Gamma}\right],
$$

where $\delta \omega\left[\Lambda, R, \Gamma, P_{\Lambda}, P_{\Gamma}\right]$ is a well defined functional, which is also an exact form. This equality shows that the difference between the Liouville form of $\left\{R, \Lambda, \Gamma ; P_{R}, P_{\Lambda}, P_{\Gamma}\right\}$ and the Liouville form of $\left\{\mathrm{R}, M, Q ; P_{\mathrm{R}}, P_{M}, P_{Q}\right\}$ is an exact form, which implies that the transformation of variables given by the set of equations (83) is canonical.

Armed with the certainty of the canonicity of the new variables, we can write the asymptotic form of the canonical variables and of the metric function $F(t, r)$. These are, for $r \rightarrow 0$

$$
\begin{aligned}
F(t, r) & =4 R_{2}^{2} \Lambda_{0}^{-2} r^{2}+O\left(r^{4}\right), \\
\mathrm{R}(t, r) & =R_{0}+R_{2} r^{2}+O\left(r^{4}\right), \\
M(t, r) & =M_{0}+M_{2} r^{2}+O\left(r^{4}\right), \\
Q(t, r) & =Q_{0}+Q_{2} r^{2}+O\left(r^{4}\right), \\
P_{\mathrm{R}}(t, r) & =O(r), \\
P_{M}(t, r) & =O(r), \\
P_{Q}(t, r) & =O(r),
\end{aligned}
$$

with

$$
\begin{aligned}
& M_{0}=l^{-2} R_{0}^{2}-\pi Q_{0}^{2} \ln \left(\frac{R_{0}}{R_{*}}\right) \\
& M_{2}=2 l^{-2} R_{0} R_{2}-2 \pi \ln \left(\frac{R_{0}}{R_{*}}\right) Q_{0} Q_{2}-\pi Q_{0}^{2} R_{2} R_{0}^{-1}-4 \Lambda_{0}^{-2} R_{2}^{2} .
\end{aligned}
$$


For $r \rightarrow \infty$, we have

$$
\begin{aligned}
F(t, r) & =2 \lambda^{2} r^{2}-2(\eta(t)+2 \rho(t))+O^{\infty}\left(r^{-2}\right), \\
\mathrm{R}(t, r) & =r+\left(2 \lambda^{2}\right)^{-1} \rho(t) r^{-1}+O^{\infty}\left(r^{-3}\right), \\
M(t, r) & =M_{+}(t)+O^{\infty}\left(r^{-2}\right) \\
Q(t, r) & =Q_{+}(t)+O^{\infty}\left(r^{-2}\right), \\
P_{\mathrm{R}}(t, r) & =O^{\infty}\left(r^{-4}\right) \\
P_{M}(t, r) & =O^{\infty}\left(r^{-5}\right), \\
P_{Q}(t, r) & =O^{\infty}\left(r^{-2}\right),
\end{aligned}
$$

where $M_{+}(t)=2(\eta(t)+2 \rho(t))$, as seen before in the surface terms (see Eq. (60) ). Note here that without the treatment of the divergence in subsection II the function $M(t, r)$ would diverge.

We are now almost ready to write the action with the new canonical variables. It is now necessary to determine the new Lagrange multipliers. In order to write the new constraints with the new Lagrange multipliers, we can use the identity given by the space derivative of $M$,

$$
M^{\prime}=-\Lambda^{-1}\left(R^{\prime} H+\frac{1}{2} P_{\Lambda}\left(H_{r}-\Gamma G\right)-\frac{1}{2 \pi} P_{\Gamma} G \ln \left(\frac{R}{R_{*}}\right) \Lambda\right) .
$$

Solving for $H$ and making use of the inverse transformations of $\Lambda$ and $P_{\Lambda}$, in Eqs. (1ㅣ) and (2), we get

$$
\begin{aligned}
H & =-\frac{M^{\prime} F^{-1} \mathrm{R}^{\prime}+F P_{M} P_{\mathrm{R}}+2 \pi Q Q^{\prime} R^{\prime} F^{-1} \ln \left(\frac{R}{R_{*}}\right)}{\left(F^{-1}\left(\mathrm{R}^{\prime}\right)^{2}-F P_{M}^{2}\right)^{\frac{1}{2}}}, \\
H_{r} & =P_{M} M^{\prime}+P_{\mathrm{R}^{\prime}} \mathrm{R}^{\prime}+P_{Q} Q^{\prime} \\
G & =-2 \pi Q^{\prime} .
\end{aligned}
$$

Following Kuchař [10], the new set of constraints, totally equivalent to the old set $H(t, r)=0, H_{r}(t, r)=0$, and $G=0$, outside the horizon points, is $M^{\prime}(t, r)=0, P_{\mathrm{R}}(t, r)=0$, and $Q^{\prime}(t, r)=0$. By continuity, this also applies on the horizon, where $F(t, r)=0$. So we can say that the equivalence is valid everywhere. The new Hamiltonian, which is the total sum of the constraints, can now be written as

$$
N H+N^{r} H_{r}+\tilde{\Phi} G=N^{M} M^{\prime}+N^{\mathrm{R}} P_{\mathrm{R}}+N^{Q} Q^{\prime}
$$

In order to determine the new Lagrange multipliers, one has to write the left hand side of the previous equation, Eq. (106), and replace the constraints on that side by their expressions as functions of the new canonical coordinates, spelt out in Eqs. (103)-(105). After manipulation, one gets

$$
\begin{aligned}
& N^{M}=-\frac{N F^{-1} R^{\prime}}{\left(F^{-1}\left(\mathrm{R}^{\prime}\right)^{2}-F P_{M}^{2}\right)^{\frac{1}{2}}}+N^{r} P_{M}, \\
& N^{\mathrm{R}}=-\frac{N F P_{M}}{\left(F^{-1}\left(\mathrm{R}^{\prime}\right)^{2}-F P_{M}^{2}\right)^{\frac{1}{2}}}+N^{r} R^{\prime}, \\
& N^{Q}=\frac{2 \pi N Q R^{\prime} F^{-1} \ln \left(\frac{R}{R_{*}}\right)}{\left(F^{-1}\left(\mathrm{R}^{\prime}\right)^{2}-F P_{M}^{2}\right)^{\frac{1}{2}}}+N^{r} P_{Q}-2 \pi \tilde{\Phi} .
\end{aligned}
$$

Using the inverse transformations Eqs. (81)-(82), and the identity $R=\mathrm{R}$, with $P_{\Gamma}=2 \pi Q$, we can write the new multipliers as functions of the old variables

$$
\begin{aligned}
& N^{M}=-N F^{-1} R^{\prime} \Lambda^{-1}+\frac{1}{2} N^{r} F^{-1} \Lambda P_{\Lambda}, \\
& N^{\mathrm{R}}=-\frac{1}{2} N P_{\Lambda}+N^{r} R^{\prime}, \\
& N^{Q}=N P_{\Gamma} R^{\prime} F^{-1} \Lambda^{-1} \ln \left(\frac{R}{R_{*}}\right)-2 \pi N^{r} \Gamma+\frac{1}{2} N^{r} P_{\Gamma} F^{-1} \ln \left(\frac{R}{R_{*}}\right) \Lambda P_{\Lambda}-2 \pi \tilde{\Phi},
\end{aligned}
$$


allowing us to determine its asymptotic conditions from the original conditions given above. These transformations are non-singular for $r>0$. As before, for $r \rightarrow 0$,

$$
\begin{aligned}
& N^{M}(t, r)=-\frac{1}{2} N_{1}(t) \Lambda_{0} R_{2}^{-1}+O\left(r^{2}\right), \\
& N^{\mathrm{R}}(t, r)=O\left(r^{4}\right), \\
& N^{Q}(t, r)=\frac{1}{2} R_{2}^{-1} \ln \left(\frac{R_{0}}{R_{*}}\right) Q_{0} \Lambda_{0} N_{1}(t)-2 \pi \tilde{\Phi}_{0}(t)+O\left(r^{2}\right),
\end{aligned}
$$

and for $r \rightarrow \infty$ we have

$$
\begin{aligned}
N^{M}(t, r) & =-\tilde{N}_{+}(t)+O^{\infty}\left(r^{-2}\right), \\
N^{\mathrm{R}}(t, r) & =O^{\infty}\left(r^{-1}\right), \\
N^{Q}(t, r) & =-2 \pi \tilde{\Phi}_{+}(t)+O^{\infty}\left(r^{-1}\right) .
\end{aligned}
$$

The conditions (113)-(118) show that the transformations in Eqs. (110)-(111) are satisfactory in the case of $r \rightarrow \infty$, but not for $r \rightarrow 0$. This is due to fact that in order to fix the Lagrange multipliers for $r \rightarrow \infty$, as we are free to do, we fix $\tilde{N}_{+}(t)$, which we already do when adding the surface term

$$
-\int d t \tilde{N}_{+} M_{+}
$$

to the action, in order to obtain the equations of motion in the bulk, without surface terms. The same is true for $\tilde{\Phi}_{+}$. However, at $r=0$, we see that fixing the multiplier $N^{M}$ to values independent of the canonical variables is not the same as fixing $N_{1} \Lambda_{0}^{-1}$ to values independent of the canonical variables. The same is true of the fixation of $N^{Q}$ with respect to $\tilde{\Phi}_{0}$. We need to rewrite the multipliers $N^{M}$ and $N^{Q}$ for the asymptotic regime $r \rightarrow 0$ without affecting their behavior for $r \rightarrow \infty$. In order to proceed we have to make one assumption, which is that the expression given in the asymptotic condition of $M(t, r)$, as $r \rightarrow 0$, for the term of order zero, $M_{0} \equiv l^{-2} R_{0}^{2}-\pi Q_{0}^{2} \ln \left(\frac{R_{0}}{R_{*}}\right)$, defines $R_{0}$ as a function of $M_{0}$ and $Q_{0}$, and $R_{0}$ is the horizon radius function, $R_{0} \equiv R_{\mathrm{h}}\left(M_{0}, Q_{0}\right)$. Also, we assume that $M_{0}>M_{\text {crit }}\left(Q_{0}\right)$, where

$$
M_{\text {crit }}\left(Q_{0}\right)=\frac{\pi Q_{0}^{2}}{2}\left(1-\ln \left(\frac{\pi Q_{0}^{2}}{2}\right)\right) .
$$

With these assumptions, we are working in the domain of the classical solutions. We can immediately obtain that the variation of $R_{0}$ is given in relation to the variations of $M_{0}$ and $Q_{0}$ as

$$
\delta R_{0}=\left(2 l^{-2} R_{0}-\pi Q_{0}^{2} R_{0}^{-1}\right)^{-1}\left(\delta M_{0}+2 \pi Q_{0} \ln \left(\frac{R_{0}}{R_{*}}\right) \delta Q_{0}\right)
$$

This expression will be used when we derive the equations of motion from the new action. We now define the new multipliers $\tilde{N}^{M}$ and $\tilde{N}^{Q}$ as

$$
\begin{aligned}
& \tilde{N}^{M}=-N^{M}\left[(1-g)+2 g\left(2 l^{-2} R_{0}-\pi Q_{0}^{2} R_{0}^{-1}\right)^{-1}\right]^{-1}, \\
& \tilde{N}^{Q}=\tilde{N}^{M} 2 g Q_{0} \ln \left(\frac{R_{0}}{R_{*}}\right)\left(2 l^{-2} R_{0}-\pi Q_{0}^{2} R_{0}^{-1}\right)^{-1}-N^{Q},
\end{aligned}
$$

where $g(r)=1+O\left(r^{2}\right)$ for $r \rightarrow 0$ and $g(r)=O^{\infty}\left(r^{-5}\right)$ for $r \rightarrow \infty$. The new multipliers, functions of the old multipliers $N^{M}$ and $N^{Q}$, have as their properties for $r \rightarrow 0$

$$
\begin{aligned}
\tilde{N}^{M}(t, r) & =\tilde{N}_{0}^{M}(t)+O\left(r^{2}\right), \\
\tilde{N}^{Q}(t, r) & =2 \pi \tilde{\Phi}_{0}(t)+O\left(r^{2}\right),
\end{aligned}
$$

and as their properties for $r \rightarrow \infty$

$$
\begin{aligned}
\tilde{N}^{M}(t, r) & =\tilde{N}_{+}(t)+O^{\infty}\left(r^{-2}\right) \\
\tilde{N}^{Q}(t, r) & =2 \pi \tilde{\Phi}_{+}(t)+O^{\infty}\left(r^{-1}\right)
\end{aligned}
$$


When the constraints $M^{\prime}=0=Q^{\prime}$ hold, $\tilde{N}_{0}^{M}$ is given by

$$
\tilde{N}_{0}^{M}=N_{1} \Lambda_{0}^{-1} .
$$

With this new constraint $\tilde{N}^{M}$, fixing $N_{1} \Lambda_{0}^{-1}$ at $r=0$ or fixing $\tilde{N}_{0}^{M}$ is equivalent, there being no problems with $N^{\mathrm{R}}$, which is left as determined in Eq. (108). With respect to $\tilde{N}^{Q}$ the same happens, i. e., fixing the zero order term of the expansion of $N^{Q}$ for $r \rightarrow 0$ is the same as fixing $\tilde{\Phi}_{0}$. At infinity there were no initial problems with the definitions of both $\tilde{N}^{M}$ and $\tilde{N}^{Q}$.

The new action is now written as the sum of $S_{\Sigma}$, the bulk action, and $S_{\partial \Sigma}$, the surface action,

$$
\begin{aligned}
S\left[M, \mathrm{R}, Q, P_{M}, P_{\mathrm{R}}, P_{Q} ; \tilde{N}^{M}, N^{\mathrm{R}}, \tilde{N}^{Q}\right]= & \int d t \int_{0}^{\infty} d r\left(P_{M} \dot{M}+P_{\mathrm{R}} \dot{\mathrm{R}}+P_{Q} \dot{Q}\right. \\
& +\tilde{N}^{Q} Q^{\prime}-N^{\mathrm{R}} P_{\mathrm{R}}+\tilde{N}^{M}(1-g) M^{\prime} \\
& +\tilde{N}^{M} 2 g\left(2 l^{-2} R_{0}-\pi Q_{0}^{2} R_{0}^{-1}\right)^{-1}\left(M^{\prime}-Q^{\prime} Q_{0} \ln \left(\frac{R_{0}}{R_{*}}\right)\right) \\
& +\int d t\left\{\left(2 R_{0} \tilde{N}_{0}^{M}-\tilde{N}_{+} M_{+}\right)+2 \pi\left(\tilde{\Phi}_{0} Q_{0}-\tilde{\Phi}_{+} Q_{+}\right)\right\} .
\end{aligned}
$$

The new equations of motion are now

$$
\begin{aligned}
\dot{M} & =0, \\
\dot{\mathrm{R}} & =N^{\mathrm{R}}, \\
\dot{Q} & =0, \\
\dot{P}_{M} & =\left(N^{M}\right)^{\prime}, \\
\dot{P}_{\mathrm{R}} & =0, \\
\dot{P}_{Q} & =\left(N^{Q}\right)^{\prime}, \\
M^{\prime} & =0, \\
P_{\mathrm{R}} & =0, \\
Q^{\prime} & =0 .
\end{aligned}
$$

where we understood $N^{M}$ to be a function of the new constraint, defined through Eq. (122) and $N^{Q}$ as the function of the new constraint defined through Eq. (123). The resulting boundary terms of the variation of this new action, Eq. (129), are, first, terms proportional to $\delta M, \delta \mathrm{R}$, and $\delta Q$ on the initial and final hypersurfaces, and, second,

$$
\int d t\left(2 R_{0} \delta \tilde{N}_{0}^{M}-M_{+} \delta \tilde{N}_{+}\right)+2 \pi\left(Q_{0} \delta \tilde{\Phi}_{0}-Q_{+} \delta \tilde{\Phi}_{+}\right) .
$$

To arrive at (139) we have used the expression in Eq. (121). The action in Eq. (129) yields the equations of motion, Eqs. (130)-(138), provided that we fix the initial and final values of the new canonical variables and that we also fix the values of $\tilde{N}_{0}^{M}$ and of $\tilde{N}_{+}$, and of $\tilde{\Phi}_{0}$ and $\tilde{\Phi}_{+}$. Thanks to the redefinition of the Lagrange multiplier, from $N^{M}$ to $\tilde{N}^{M}$, the fixation of those quantities, $\tilde{N}_{0}^{M}$ and $\tilde{N}_{+}$, has the same meaning it had before the canonical transformations and the redefinition of $N^{M}$. The same happens with $\tilde{\Phi}_{0}$ and $\tilde{\Phi}_{+}$. This keeping of meaning is guaranteed through the use of our gauge freedom to choose the multipliers, and at the same time not fixing the boundary variations independently of the choice of Lagrange multipliers, which in turn allow us to have a well defined variational principle for the action.

\section{Hamiltonian reduction}

We now solve the constraints in order to reduce to the true dynamical degrees of freedom. The equations of motion (130)-(138) allow us to write $M$ and $Q$ as independent functions of space, $r$,

$$
\begin{aligned}
M(t, r) & =\mathbf{m}(t), \\
Q(t, r) & =\mathbf{q}(t) .
\end{aligned}
$$


The reduced action, with the constraints taken into account, is then

$$
S\left[\mathbf{m}, \mathbf{p}_{\mathbf{m}}, \mathbf{q}, \mathbf{p}_{\mathbf{q}} ; \tilde{N}_{0}^{M}, \tilde{N}_{+}, \tilde{\Phi}_{0}, \tilde{\Phi}_{+}\right]=\int d t \mathbf{p}_{\mathbf{m}} \dot{\mathbf{m}}+\mathbf{p}_{\mathbf{q}} \dot{\mathbf{q}}-\mathbf{h},
$$

where

$$
\begin{aligned}
\mathbf{p}_{\mathbf{m}} & =\int_{0}^{\infty} d r P_{M}, \\
\mathbf{p}_{\mathbf{q}} & =\int_{0}^{\infty} d r P_{Q},
\end{aligned}
$$

and the reduced Hamiltonian, $\mathbf{h}$, is now written as

$$
\mathbf{h}(\mathbf{m}, \mathbf{q} ; t)=-2 R_{\mathrm{h}} \tilde{N}_{0}^{M}+\tilde{N}_{+} \mathbf{m}+2 \pi \mathbf{q}\left(\tilde{\Phi}_{+}-\tilde{\Phi}_{0}\right),
$$

with $R_{\mathrm{h}}$ being the horizon radius. We also have that $\mathbf{m}>M_{\text {crit }}(\mathbf{q})$, according to the assumptions made in the previous subsection. Thanks to the functions $\tilde{N}_{0}^{M}(t), \tilde{N}_{+}(t), \tilde{\Phi}_{0}(t)$, and $\tilde{\Phi}_{+}(t)$ the Hamiltonian $\mathbf{h}$ is an explicitly time dependent function. The variational principle associated with the reduced action, Eq. (142), will fix the values of $\mathbf{m}$ and $\mathbf{q}$ on the initial and final hypersurfaces, or in the spirit of the classical analytical mechanics, the Hamiltonian principle fixes the initial and final values of the canonical coordinates. The equations of motion are

$$
\begin{aligned}
\dot{\mathbf{m}} & =0 \\
\dot{\mathbf{q}} & =0 \\
\dot{\mathbf{p}}_{\mathbf{m}} & =2 \tilde{N}_{0}^{M}\left(2 l^{-2} R_{\mathrm{h}}-\pi Q_{0}^{2} R_{\mathrm{h}}^{-1}\right)^{-1}-\tilde{N}_{+} \\
\dot{\mathbf{p}}_{\mathbf{q}} & =2 \pi\left\{2 \mathbf{q} \ln \left(\frac{R_{\mathrm{h}}}{R_{*}}\right)\left(2 l^{-2} R_{\mathrm{h}}-\pi Q_{0}^{2} R_{\mathrm{h}}^{-1}\right)^{-1} \tilde{N}_{0}^{M}+\tilde{\Phi}_{0}-\tilde{\Phi}_{+}\right\} .
\end{aligned}
$$

The equation of motion for $\mathbf{m}$, Eq. (146), is understood as saying that $\mathbf{m}$ is, on a classical solution, equal to the mass parameter $M$ of the solution, Eq. (24). The same goes for the function $\mathbf{q}$, where Eq. (147) implies that $\mathbf{q}$ is equal to the charge parameter $Q$ on a classical solution, Eq. (24). In order to interpret the other equation of motion, Eq. (148), we have to recall that from Eq. (78) one has $P_{M}=-T^{\prime}$, where $T$ is the Killing time. This, together with the definition of $\mathbf{p}_{\mathbf{m}}$, given in Eq. (143), yields

$$
\mathbf{p}_{\mathbf{m}}=T_{0}-T_{+}
$$

where $T_{0}$ is the value of the Killing time at the left end of the hypersurface of a certain $t$, and $T_{+}$is the Killing time at spatial infinity, the right end of the same hypersurface of $t$. As the hypersurface evolves in the spacetime of the black hole solution, the right hand side of Eq. (147) is equal to $\dot{T}_{0}-\dot{T}_{+}$. Finally, after the definition

$$
\mathbf{p}_{\mathbf{q}}=\xi_{0}-\xi_{+}
$$

obtained from Eqs. (64), (80), and (144), Eq. (149) gives in the right hand side $\dot{\xi}_{0}-\dot{\xi}_{+}$, which is the diference of the time derivatives of the electromagnetic gauge $\xi(t, r)$ at $r=0$ and at infinity.

\section{E. Quantum theory and partition function}

The next step is to quantize the reduced Hamiltonian theory, by building the time evolution operator quantum mechanically and then obtaining a partition function through the analytic continuation of the same operator [1][6]. The variables $\mathbf{m}$ and $\mathbf{q}$ are regarded here as configuration variables. These variables satisfy the inequality $\mathbf{m}>M_{\text {crit }}(\mathbf{q})$. The wave functions will be of the form $\psi(\mathbf{m}, \mathbf{q})$, with the inner product given by

$$
(\psi, \chi)=\int_{A} \mu d \mathbf{m} d \mathbf{q} \bar{\psi} \chi
$$

where $A$ is the domain of integration defined by $\mathbf{m}>M_{\text {crit }}(\mathbf{q})$ and $\mu(\mathbf{m}, \mathbf{q})$ is a smooth and positive weight factor for the integration measure. It is assumed that $\mu$ is a slow varying function, otherwise arbitrary. We are thus working in the Hilbert space defined as $\mathscr{H}:=L^{2}(A ; \mu d \mathbf{m} d \mathbf{q})$. 
The Hamiltonian operator, written as $\hat{\mathbf{h}}(t)$, acts through pointwise multiplication by the function $\mathbf{h}(\mathbf{m}, \mathbf{q} ; t)$, which on a function of our working Hilbert space reads

$$
\hat{\mathbf{h}}(t) \psi(\mathbf{m}, \mathbf{q})=\mathbf{h}(\mathbf{m}, \mathbf{q} ; t) \psi(\mathbf{m}, \mathbf{q}) .
$$

This Hamiltonian operator is an unbounded essentially self-adjoint operator. The corresponding time evolution operator in the same Hilbert space, which is unitary due to the fact that the Hamiltonian operator is self-adjoint, is

$$
\hat{K}\left(t_{2} ; t_{1}\right)=\exp \left[-i \int_{t_{1}}^{t_{2}} d t^{\prime} \hat{\mathbf{h}}\left(t^{\prime}\right)\right] .
$$

This operator acts also by pointwise multiplication in the Hilbert space. We now define

$$
\begin{aligned}
\mathcal{T} & :=\int_{t_{1}}^{t_{2}} d t \tilde{N}_{+}(t), \\
\Theta & :=\int_{t_{1}}^{t_{2}} d t \tilde{N}_{0}^{M}(t), \\
\Xi_{0} & :=\int_{t_{1}}^{t_{2}} d t \tilde{\Phi}_{0}(t), \\
\Xi_{+} & :=\int_{t_{1}}^{t_{2}} d t \tilde{\Phi}_{+}(t) .
\end{aligned}
$$

Using (145), (154)-(158) we write the function $K$, which is in fact the action of the operator in the Hilbert space, as

$$
K\left(\mathbf{m} ; \mathcal{T}, \Theta, \Xi_{0}, \Xi_{+}\right)=\exp \left[-i \mathbf{m} \mathcal{T}+2 i R_{\mathrm{h}} \Theta-2 \pi i \mathbf{q}\left(\Xi_{+}-\Xi_{0}\right)\right] .
$$

This expression indicates that $\hat{K}\left(t_{2} ; t_{1}\right)$ depends on $t_{1}$ and $t_{2}$ only through the functions $\mathcal{T}, \Theta, \Xi_{0}$, and $\Xi_{+}$. Thus, the operator corresponding to the function $K$ can now be written as $\hat{K}\left(\mathcal{T}, \Theta, \Xi_{0}, \Xi_{+}\right)$. The composition law in time $\hat{K}\left(t_{3} ; t_{2}\right) \hat{K}\left(t_{2} ; t_{1}\right)=\hat{K}\left(t_{3} ; t_{1}\right)$ can be regarded as a sum of the parameters $\mathcal{T}, \Theta, \Xi_{0}$, and $\Xi_{+}$inside the operator $\hat{K}\left(\mathcal{T}, \Theta, \Xi_{0}, \Xi_{+}\right)$. These parameters are evolutions parameters defined by the boundary conditions, i.e., $\mathcal{T}$ is the Killing time elapsed at right spatial infinity and $\Theta$ is the boost parameter elapsed at the bifurcation circle; $\Xi_{0}$ and $\Xi_{+}$are line integrals along timelike curves of constant $r$, and constant angular variables, at $r=0$ and at infinity.

\section{F. Thermodynamics}

We can now build the partition function for this system. The path to follow is to continue the operator to imaginary time and take the trace over a complete orthogonal basis. Our classical thermodynamic situation consists of a threedimensional spherically symmetric charged black hole, asymptotically anti-de Sitter, in thermal equilibrium with a bath of Hawking radiation. Ignoring back reaction from the radiation, the geometry is described by the solutions in Eqs. (24)-(26). Thus, we consider a thermodynamic ensemble in which the temperature, or more appropriately here, the inverse temperature $\beta$, and the electric potential $\bar{\phi}$ are fixed. This characterizes a grand canonical ensemble, and the partition function $\mathcal{Z}(\beta, \bar{\phi})$ arises naturally in such an ensemble. To analytically continue the Lorentzian solution we put $\mathcal{T}=-i \beta$, and $\Theta-2 \pi i$, this latter choice based on the regularity of the classical Euclidean solution. We also choose $\Xi_{0}=0$ and $\Xi_{+}=i \beta \bar{\phi}$.

We arrive then at the following expression for the partition function

$$
\mathcal{Z}(\beta, \bar{\phi})=\operatorname{Tr}[\hat{K}(-i \beta,-2 \pi i, 0, i \beta \bar{\phi})] .
$$

From Eq. (159) this is realized as

$$
\mathcal{Z}(\beta . \bar{\phi})=\int_{A} \mu d \mathbf{m} d \mathbf{q} \exp \left[-\beta(\mathbf{m}-2 \pi \mathbf{q} \bar{\phi})+4 \pi R_{\mathrm{h}}\right]\langle\mathbf{m} \mid \mathbf{m}\rangle .
$$

Since $\langle\mathbf{m} \mid \mathbf{m}\rangle$ is equal to $\delta(0)$, one has to regularize Eq. (161). Following the procedure developed in the Louko-Whiting approach [2], this means regularizing and normalizing the operator $\hat{K}$ beforehand. This leads to

$$
\mathcal{Z}_{\mathrm{ren}}(\beta, \bar{\phi})=\mathcal{N} \int_{A} \mu d \mathbf{m} d \mathbf{q} \exp \left[-\beta(\mathbf{m}-2 \pi \mathbf{q} \bar{\phi})+4 \pi R_{\mathrm{h}}\right]
$$


where $\mathcal{N}$ is a normalization factor and $A$ is the domain of integration. Provided the weight factor $\mu$ is slowly varying compared to the exponential in Eq. (162), and using the fact that the horizon radius $R_{\mathrm{h}}$ is a function of $\mathbf{m}$ and $\mathbf{q}$, the integral in Eq. (162) is convergent. Changing integration variables, from $\mathbf{m}$ to $R_{\mathrm{h}}$, keeping $\mathbf{q}$, where

$$
\mathbf{m}=l^{-2} R_{\mathrm{h}}^{2}-\pi \mathbf{q}^{2} \ln \left(\frac{R_{\mathrm{h}}}{R_{*}}\right)
$$

the integral Eq. (162) becomes

$$
\mathcal{Z}_{\text {ren }}(\beta, \bar{\phi})=\mathcal{N} \int_{A^{\prime}} \tilde{\mu} d R_{\mathrm{h}} d \mathbf{q} \exp \left(-I_{*}\right)
$$

where $A^{\prime}$ is new the domain of integration after changing variables, and the function $I_{*}\left(R_{\mathrm{h}}\right)$, a kind of an effective action (see [16]), is written as

$$
I_{*}\left(R_{\mathrm{h}}\right):=\beta\left(l^{-2} R_{\mathrm{h}}^{2}-\pi \mathbf{q}^{2} \ln \left(\frac{R_{\mathrm{h}}}{R_{*}}\right)-2 \pi \mathbf{q} \bar{\phi}\right)-4 \pi R_{\mathrm{h}} .
$$

The new domain of integration, $A^{\prime}$, is defined by the inequalities $0 \leq R_{\mathrm{h}}$ and $\mathbf{q}^{2} \leq 2 R_{\mathrm{h}}^{2} \pi^{-1} l^{-2}$. The new weight factor $\widetilde{\mu}$ includes the Jacobian of the transformation. Since the weight factor $\widetilde{\mu}$ is slowly varying, we can estimate the integral of $\mathcal{Z}_{\text {ren }}(\beta, \bar{\phi})$ by the saddle point approximation. For that we have to calculate the critical points. Firstly one finds the value of $\mathbf{q}$ for which

$$
\frac{\partial I_{*}\left(R_{\mathrm{h}}, \mathbf{q}\right)}{\partial \mathbf{q}}=0
$$

The value is

$$
\mathbf{q}^{*}=-\bar{\phi}\left[\ln \left(\frac{R_{\mathrm{h}}}{R_{*}}\right)\right]^{-1}
$$

Replacing this value in (165), we obtain

$$
I_{*}\left(R_{\mathrm{h}}, \mathbf{q}^{*}\left(R_{\mathrm{h}}\right)\right)=\beta\left(l^{-2} R_{\mathrm{h}}^{2}+\pi \bar{\phi}^{2}\left[\ln \left(\frac{R_{\mathrm{h}}}{R_{*}}\right)\right]^{-1}\right)-4 \pi R_{\mathrm{h}} .
$$

Deriving expression (168) with respect to $R_{\mathrm{h}}$ and making it zero, we obtain

$$
\frac{\partial I_{*}\left(R_{\mathrm{h}}\right)}{\partial R_{\mathrm{h}}}=2 \beta l^{-2} R_{\mathrm{h}}-\beta \bar{\phi}^{2}\left[\ln \left(\frac{R_{\mathrm{h}}}{R_{*}}\right)\right]^{-2} R_{\mathrm{h}}^{-1}-4 \pi=0,
$$

which implies

$$
2 \beta l^{-2} R_{\mathrm{h}}^{2}-\beta \bar{\phi}^{2}\left[\ln \left(\frac{R_{\mathrm{h}}}{R_{*}}\right)\right]^{-2}-4 \pi R_{\mathrm{h}}=0 .
$$

The solutions $R_{\mathrm{h}}^{*}$ to the equation (170) gives us the pair $\left(R_{\mathrm{h}}^{*}, \mathbf{q}^{*}\right)$ of critical points, with $\mathbf{q}^{*}$ given in Eq. (167), where $R_{\mathrm{h}}=R_{\mathrm{h}}^{*}$. Of these, the classical solution comes from the one critical pair that is also the global minimum of the effective action $I_{*}$, denoted by $\left(R_{\mathrm{h}}^{+}, \mathbf{q}^{+}\right)$, depending on the interval of values to which $\bar{\phi}$ belongs. The renormalized partition function is then

$$
\mathcal{Z}_{\mathrm{ren}}(\beta, \bar{\phi})=\mathrm{P} \exp \left[-\beta\left(l^{-2}\left(R_{\mathrm{h}}^{+}\right)^{2}-\pi\left(\mathbf{q}^{+}\right)^{2} \ln \left(\frac{R_{\mathrm{h}}^{+}}{R_{*}}\right)-2 \pi \mathbf{q}^{+} \bar{\phi}\right)+4 \pi R_{\mathrm{h}}^{+}\right],
$$

where $\mathrm{P}$ is a slowly varying prefactor and $\left(R_{\mathrm{h}}^{+}, \mathbf{q}^{+}\right)$is the global minimum of the effective action (165). In the domain of integration the dominating contribution comes from the vicinity of $R_{\mathrm{h}}=R_{\mathrm{h}}^{+}$. We now write the logarithm of $\mathcal{Z}_{\text {ren }}$ as

$$
\ln \left(\mathcal{Z}_{\text {ren }}\right)=\ln \mathrm{P}-\beta\left(l^{-2}\left(R_{\mathrm{h}}^{+}\right)^{2}-\pi\left(\mathbf{q}^{+}\right)^{2} \ln \left(\frac{R_{\mathrm{h}}^{+}}{R_{*}}\right)-2 \pi \mathbf{q}^{+} \bar{\phi}\right)+4 \pi R_{\mathrm{h}}^{+} .
$$


By ignoring the prefactor's logarithm, which closer to $R_{\mathrm{h}}^{+}$is less relevant, we are able to determine the value of $\mathbf{m}$ at the critical point, where we find that it corresponds to the value of the mass of the classical solutions of the black holes (see Eq. (24)). Thus, when the critical point dominates the partition function, we have that the mean energy $\langle E\rangle$ is given by

$$
\langle E\rangle=-\frac{\partial}{\partial \beta} \ln \mathcal{Z}_{\text {ren }} \approx 2 \lambda^{2}\left(R_{\mathrm{h}}^{+}\right)^{2}-\pi\left(\mathbf{q}^{+}\right)^{2} \ln \left(\frac{R_{\mathrm{h}}^{+}}{R_{*}}\right)=\mathbf{m}^{+},
$$

where $\mathbf{m}^{+}$is obtained from Eq. (163) evaluated at $R_{\mathrm{h}}^{+}$. The thermal expectation value of the charge is

$$
\langle Q\rangle=\beta^{-1} \frac{\partial}{\partial \bar{\phi}} \ln \mathcal{Z}_{\text {ren }} \approx 2 \pi \mathbf{q}^{+}
$$

The temperature of the black hole, $\mathbf{T} \equiv \beta^{-1}$, is

$$
\mathbf{T}=\frac{1}{4 \pi}\left(4 \lambda^{2}\left(R_{\mathrm{h}}^{+}\right)-\pi\left(\mathbf{q}^{+}\right)^{2}\left(R_{\mathrm{h}}^{+}\right)^{-1}\right)
$$

where $\mathbf{q}^{+}$is the function in (167) evaluated at $R_{\mathrm{h}}^{+}$. If the maximum value of the charge $\mathbf{q}^{+}$is chosen, i.e., $\mathbf{q}^{2}=$ $2 R_{\mathrm{h}}^{2} \pi^{-1} l^{-2}$, which is the value of the charge of the extreme solution of the black hole, and is replaced into (175), then the temperature is null, as expected from an extreme solution. It can be shown that $\partial \mathbf{m}^{+} / \partial \beta<0$, which through the constant $\bar{\phi}$ heat capacity $C_{\bar{\phi}}=-\beta^{2}(\partial\langle E\rangle / \partial \beta)$ tells us that the system is thermodynamically stable. The entropy is given by

$$
S=\left(1-\beta \frac{\partial}{\partial \beta}\right)\left(\ln \mathcal{Z}_{\text {ren }}\right) \approx 4 \pi R_{\mathrm{h}}^{+} .
$$

This is the entropy of the charged BTZ black hole [20, 23] (see also [28]). This entropy includes the extreme solution, as the specific value of the charge is irrelevant for the determination of the entropy, provided we are in the domain of validity of the approximation and $\mathbf{q}^{2} \leq 2 R_{\mathrm{h}}^{2} \pi^{-1} l^{-2}$.

\section{HAMILTONIAN THERMODYNAMICS OF THE GENERAL RELATIVISTIC CYLINDRICAL DIMENSIONALLY REDUCED CHARGED BLACK HOLE $(\omega=0)$}

\section{A. The metric, the scalar $\phi$, and the vector potential one-form}

For $\omega=0$, the corresponding three-dimensional charged Brans-Dicke theory is obtained from the cylindrical dimensionally reduced black hole of four-dimensional general relativity, with a Maxwell term [21, 22, 25]. Then general metric in Eq. (2), the $\phi$ field in Eq. (6), and the vector potential one-form (8) reduce to the following

$$
\begin{aligned}
d s^{2} & =-\left[(a R)^{2}-\frac{M}{2(a R)}+\frac{Q^{2}}{16(a R)^{2}}\right] d T^{2}+\frac{d R^{2}}{(a R)^{2}-\frac{M}{2(a R)}+\frac{Q^{2}}{16(a R)^{2}}}+R^{2} d \varphi^{2}, \\
e^{-2 \phi} & =a R \\
A & =\alpha^{-1} \frac{Q}{R} d T
\end{aligned}
$$

with $M=2 b, a=\sqrt{\frac{2}{3}}|\lambda|=l^{-1}$, and $\alpha=4 a$, where the $l$ is the AdS length. The Schwarzschild coordinates are again $\{T, R, \varphi\}$. Unlike the charged BTZ solution, this solution (177)-(179) has a metric function whose mass term depends on $R$. This behavior is similar to the Reissner-Nördstrom black hole metric function [21]. In Fig. 2] we show the Carter-Penrose diagram of the black hole solution for $\omega=0$, where again $R_{\mathrm{h}}$ is the horizon radius, given by the larger positive real root of

$$
\left(a R_{\mathrm{h}}\right)^{4}-\frac{1}{2} M\left(a R_{\mathrm{h}}\right)+\left(\frac{Q}{4}\right)^{2}=0
$$

$R=0$ is the radius of the timelike curvature singularity, and $R=\infty$ is the spatial infinity. 


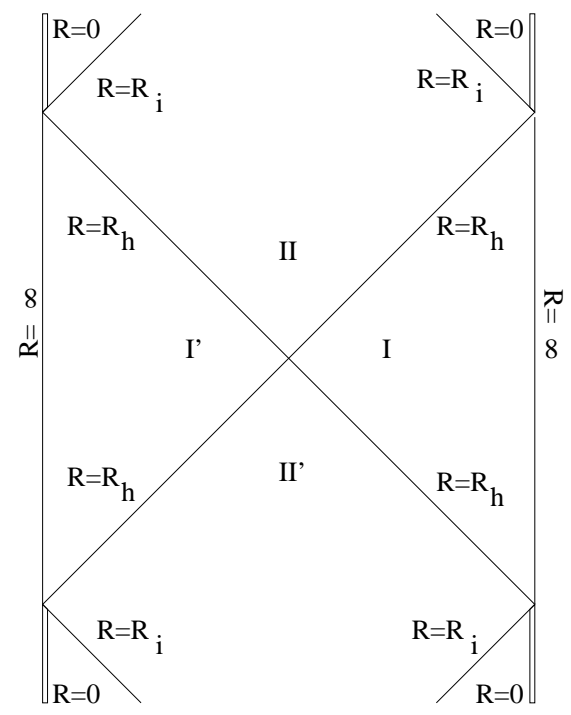

FIG. 2: The Carter-Penrose diagram for the charged $\omega=0$ case, where $R_{\mathrm{h}}$ is the outer horizon radius, $R_{\mathrm{i}}$ is the inner horizon radius, and the double line is the timelike singularity.

\section{B. Canonical formalism}

The action with $\omega=0$ becomes, following Eqs. (21)-(23), and excluding surface terms,

$$
\begin{aligned}
S\left[\Lambda, R, \Gamma, \dot{\Lambda}, \dot{R}, \dot{\Gamma} ; N, N^{r}, \Phi\right]= & \int d t \int_{0}^{\infty} d r \alpha\left\{\lambda^{2} \Lambda N R^{2}-N^{-1} R \dot{R} \dot{\Lambda}-\frac{1}{2} N^{-1} \Lambda \dot{R}^{2}+N^{-1} R \dot{R}\left(\Lambda N^{r}\right)^{\prime}\right. \\
& +N^{-1} N^{r} R R^{\prime} \dot{\Lambda}-N^{-1} N^{r} \Lambda R R^{\prime}\left(N^{r}\right)^{\prime}-N^{-1}\left(N^{r}\right)^{2} R R^{\prime} \Lambda^{\prime}+N^{-1} N^{r} \Lambda \dot{R} R^{\prime} \\
& -\frac{1}{2} N^{-1}\left(N^{r}\right)^{2} \Lambda\left(R^{\prime}\right)^{2}-\left(\Lambda^{-1}\right)^{\prime} R R^{\prime} N-\frac{1}{2} \Lambda^{-1}\left(R^{\prime}\right)^{2} N-\Lambda^{-1} R R^{\prime \prime} N \\
& \left.+\frac{1}{2} \alpha N^{-1} \Lambda^{-1} R^{2}\left(\dot{\Gamma}-\Phi^{\prime}\right)^{2}\right\}
\end{aligned}
$$

where 'means derivative with respect to time $t$ and ' is the derivative with respect to $r$, and where all the explicit functional dependences are omitted. Depending on the situation we use four different letters containing the same information but with slightly different numerical values. Thus, $a, \lambda, l$ and $\alpha$ are related by $a^{2}=\frac{2}{3} \lambda^{2}=l^{-2}$, and $\alpha=4 a$, where $l$ is the AdS length. From this action one determines the canonical momenta, conjugate to $\Lambda, R$, and $\Gamma$ respectively

$$
\begin{aligned}
& P_{\Lambda}=-\alpha N^{-1} R\left\{\dot{R}-R^{\prime} N^{r}\right\}, \\
& P_{R}=-\alpha N^{-1}\left\{R\left[\dot{\Lambda}-\left(\Lambda N^{r}\right)^{\prime}\right]+\Lambda\left[\dot{R}-N^{r} R^{\prime}\right]\right\} \\
& P_{\Gamma}=\alpha N^{-1} \Lambda^{-1} R^{2}\left(\dot{\Gamma}-\Phi^{\prime}\right) .
\end{aligned}
$$

By performing a Legendre transformation, we obtain

$$
\begin{aligned}
\mathcal{H}= & N\left\{-\alpha^{-1} R^{-1} P_{\Lambda} P_{R}+\frac{1}{2} \alpha^{-1} \Lambda R^{-2}\left(P_{\Lambda}^{2}+P_{\Gamma}^{2}\right)+\alpha \Lambda^{-1} R R^{\prime \prime}-\alpha \Lambda^{-2} R R^{\prime} \Lambda^{\prime}+\frac{1}{2} \Lambda^{-1}\left(R^{\prime}\right)^{2}-\lambda^{2} \Lambda R^{2}\right\} \\
& +N^{r}\left\{P_{R} R^{\prime}-P_{\Lambda}^{\prime} \Lambda-P_{\Gamma}^{\prime} \Gamma\right\}+\tilde{\Phi}\left\{-P_{\Gamma}^{\prime}\right\} \equiv N H+N^{r} H_{r}+\tilde{\Phi} G,
\end{aligned}
$$

with the new Lagrange multiplier being $\tilde{\Phi}:=\Phi-N^{r} \Gamma$. The action in Hamiltonian form is then

$$
S\left[\Lambda, R, \Gamma, P_{\Lambda}, P_{R}, P_{\Gamma} ; N, N^{r}, \tilde{\Phi}\right]=\int d t \int_{0}^{\infty} d r\left\{P_{\Lambda} \dot{\Lambda}+P_{R} \dot{R}+P_{\Gamma} \dot{\Gamma}-N H-N^{r} H_{r}-\tilde{\Phi} G\right\}
$$


The equations of motion are

$$
\begin{aligned}
\dot{\Lambda}= & -N \alpha^{-1} R^{-1} P_{R}+N \alpha^{-1} \Lambda R^{-2} P_{\Lambda}+N^{r} \Lambda \\
\dot{R}= & -N \alpha^{-1} P_{\Lambda} R^{-1}+N^{r} R^{\prime} \\
\dot{\Gamma}= & \alpha^{-1} N \Lambda R^{-2} P_{\Gamma}+\left(N^{r} \Gamma\right)^{\prime} \tilde{\Phi}^{\prime} \\
\dot{P_{R}}= & -N \alpha^{-1} P_{\Lambda} P_{R} R^{-2}+N \alpha^{-1} \Lambda\left(P_{\Lambda}^{2}+P_{\Gamma}^{2}\right) R^{-3}-\left((N \alpha)^{\prime} \Lambda^{-1} R\right)^{\prime}-(N \alpha)\left(\Lambda^{-1} R^{\prime}\right)^{\prime} \\
& +2 N \alpha \lambda^{2} \Lambda R+\left(N^{r} P_{R}\right)^{\prime} \\
\dot{P}_{\Lambda}= & -\frac{1}{2} N \alpha^{-1} R^{-2}\left(P_{\Lambda}^{2}+P_{\Gamma}^{2}\right)-(N \alpha)^{\prime} R R^{\prime} \Lambda^{-2}-\frac{1}{2} N \alpha\left(R^{\prime}\right)^{2} \Lambda^{-2}+N \alpha \lambda^{2} R^{2}+N^{r} P_{\Lambda}^{\prime}, \\
\dot{P}_{\Gamma}= & N^{r} P_{\Gamma}^{\prime} .
\end{aligned}
$$

In order to have a well defined variational principle, we need to eliminate the surface terms of the original bulk action, which render the original action itself ill defined for a correct determination of the equations of motion through variational methods. Through the choice of added surface terms one can achieve this elimination. The action (186) has the following extra surface terms, after variation

$$
\begin{aligned}
\text { Surface terms }= & \left\{\alpha\left(-N \Lambda^{-1} R \delta R^{\prime}+N^{\prime} \Lambda^{-1} R \delta R-N^{r} P_{R} \delta R+N^{r} \Lambda \delta P_{\Lambda}+N R R^{\prime} \Lambda^{-2} \delta \Lambda\right)\right. \\
& \left.+N^{r} \Gamma \delta P_{\Gamma}+\tilde{\Phi} \delta P_{\Gamma}\right\}\left.\right|_{0} ^{\infty} .
\end{aligned}
$$

In order to evaluate this expression, we need to know the asymptotic conditions of each of the functions of $(t, r)$.

Starting with the limit $r \rightarrow 0$ we assume

$$
\begin{aligned}
\Lambda(t, r) & =\Lambda_{0}(t)+O\left(r^{2}\right) \\
R(t, r) & =R_{0}(t)+R_{2}(t) r^{2}+O\left(r^{4}\right), \\
\Gamma(t, r) & =O(r) \\
P_{\Lambda}(t, r) & =O\left(r^{3}\right) \\
P_{R}(t, r) & =O(r) \\
P_{\Gamma}(t, r) & =Q_{0}(t)+Q_{2}(t) r^{2}+O\left(r^{4}\right), \\
N(t, r) & =N_{1}(t) r+O\left(r^{3}\right) \\
N^{r}(t, r) & =N_{1}^{r}(t) r+O\left(r^{3}\right), \\
\tilde{\Phi}(t, r) & =\tilde{\Phi}_{0}(t)+O\left(r^{2}\right) .
\end{aligned}
$$

With these conditions, we have for the surface terms at $r=0$,

$$
\text { Surface terms }\left.\right|_{r=0}=-\alpha N_{1} R_{0} \Lambda_{0}^{-1} \delta R_{0}-\tilde{\Phi}_{0} \delta Q_{0}
$$

In the same way, for $r \rightarrow \infty$,

$$
\begin{aligned}
\Lambda(t, r) & =l r^{-1}+l^{3} \eta(t) r^{-4}+O^{\infty}\left(r^{-5}\right), \\
R(t, r) & =r+l^{2} \rho(t) r^{-2}+O^{\infty}\left(r^{-3}\right), \\
\Gamma(t, r) & =O^{\infty}\left(r^{-2}\right), \\
P_{\Lambda}(t, r) & =O^{\infty}\left(r^{-2}\right), \\
P_{R}(t, r) & =O^{\infty}\left(r^{-4}\right), \\
P_{\Gamma}(t, r) & =Q_{+}(t)+O^{\infty}\left(r^{-1}\right), \\
N(t, r) & =R(t, r)^{\prime} \Lambda(t, r)^{-1}\left(\tilde{N}_{+}(t)+O^{\infty}\left(r^{-5}\right)\right), \\
N^{r}(t, r) & =O^{\infty}\left(r^{-2}\right), \\
\tilde{\Phi}(t, r) & =\tilde{\Phi}_{+}(t)+O^{\infty}\left(r^{-1}\right) .
\end{aligned}
$$

These conditions imply for the surface terms in the limit $r \rightarrow \infty$,

$$
\text { Surface terms }\left.\right|_{r \rightarrow \infty}=\alpha \delta\left(M_{+}\right) \tilde{N}_{+}+\tilde{\Phi}_{+} \delta Q_{+},
$$

where $M_{+}(t)=\alpha(\eta(t)+3 \rho(t))$. So, the surface term to be added to (186) is

$$
S_{\partial \Sigma}\left[\Lambda, R, Q_{0}, Q_{+} ; N, \tilde{\Phi}_{0}, \tilde{\Phi}_{+}\right]=\int d t\left(\frac{1}{2} \alpha R_{0}^{2} N_{1} \Lambda_{0}^{-1}-\tilde{N}_{+} M_{+}+\tilde{\Phi}_{0} Q_{0}-\tilde{\Phi}_{+} Q_{+}\right)
$$


What is left after varying this last surface term and adding it to the varied initial action (see Eq. (186)) is

$$
\int d t\left(\frac{1}{2} \alpha R_{0}^{2} \delta\left(N_{1} \Lambda_{0}^{-1}\right)-M_{+} \delta \tilde{N}_{+}+Q_{0} \delta \tilde{\Phi}_{0}-Q_{+} \delta \tilde{\Phi}_{+}\right) .
$$

We choose to fix $N_{1} \Lambda_{0}^{-1}$ and $\tilde{\Phi}_{0}$ on the horizon, and $\tilde{N}_{+}$and $\tilde{\Phi}_{+}$at infinity, which makes the surface variation (215) disappear.

\section{Reconstruction, canonical transformation, and action}

In order to reconstruct the mass and the time from the canonical data, which amounts to making a canonical transformation, we have to rewrite the general form of the solutions in Eqs. (177)-(179). However, in addition to reconstructing the mass as done in [1], we have to consider how to reconstruct the charge from the canonical data. We follow Kuchař [10] for this reconstruction. We concentrate our analysis on the right static region of the Carter-Penrose diagram.

Developing the Killing time $T$ as function of $(t, r)$ in the expression for the vector potential (179), and making use of the gauge freedom that allows us to write

$$
A=\alpha^{-1} \frac{Q}{R} d T+d \xi
$$

where $\xi(t, r)$ is an arbitrary continuous function of $t$ and $r$, we can write the one-form potential as

$$
A=\left(\alpha^{-1} \frac{Q}{R} T^{\prime}+\xi^{\prime}\right) d r+\left(\alpha^{-1} \frac{Q}{R} \dot{T}+\dot{\xi}\right) d t .
$$

Equating the expression (217) with Eq. (23), and making use of the definition of $P_{\Gamma}$ in Eq. (184), we arrive at

$$
P_{\Gamma}=Q
$$

In the right static region we define $F$ as

$$
F(R(t, r))=(a R(t, r))^{2}-\frac{M}{2 a R(t, r)}+\frac{P_{\Gamma}^{2}}{16(a R(t, r))^{2}} .
$$

Note that here, $R_{*}$, the large radius of a finite frontier, which can be used to renormalize the asymptotic properties of the future canonical coordinate $M(t, r)$ (see subsections II and III C), is not needed; in fact bringing to the $\omega=0$ solution introduces complications at the $r=0$ frontier. Thus, in this section we do not mention $R_{*}$. Then, making the following substitutions

$$
T=T(t, r), \quad R=R(t, r),
$$

in the solutions above, Eqs. (177)-(178), one has

$$
d s^{2}=-\left(F \dot{T}^{2}-F^{-1} \dot{R}^{2}\right) d t^{2}+2\left(-F T^{\prime} \dot{T}+F^{-1} R^{\prime} \dot{R}\right) d t d r+\left(-F\left(T^{\prime}\right)^{2}+F^{-1} \dot{R}^{2}\right) d r^{2}+R^{2} d \varphi^{2}
$$

This introduces the ADM foliation directly into the solutions. Comparing it with the ADM metric (21), written in another form as

$$
d s^{2}=-\left(N^{2}-\Lambda^{2}\left(N^{r}\right)^{2}\right) d t^{2}+2 \Lambda^{2} N^{r} d t d r+\Lambda^{2} d r^{2}+R^{2} d \varphi^{2}
$$

we can write a set of three equations

$$
\begin{aligned}
\Lambda^{2} & =-F\left(T^{\prime}\right)^{2}+F^{-1}\left(R^{\prime}\right)^{2}, \\
\Lambda^{2} N^{r} & =-F T^{\prime} \dot{T}+F^{-1} R^{\prime} \dot{R}, \\
N^{2}-\Lambda^{2}\left(N^{r}\right)^{2} & =F \dot{T}^{2}-F^{-1} \dot{R}^{2} .
\end{aligned}
$$

The first two equations, Eq. (223) and Eq. (224), give

$$
N^{r}=\frac{-F T^{\prime} \dot{T}+F^{-1} R^{\prime} \dot{R}}{-F\left(T^{\prime}\right)^{2}+F^{-1}\left(R^{\prime}\right)^{2}} .
$$


This one solution, together with Eq. (223), give

$$
N=\frac{R^{\prime} \dot{T}-T^{\prime} \dot{R}}{\sqrt{-F\left(T^{\prime}\right)^{2}+F^{-1}\left(R^{\prime}\right)^{2}}} .
$$

One can show that $N(t, r)$ is positive. Next, putting Eq. (226) and Eq. (227) into the definition of the conjugate momentum of the canonical coordinate $\Lambda$, given in Eq. (182), one finds the spatial derivative of $T(t, r)$ as a function of the canonical coordinates, i.e.,

$$
-T^{\prime}=\alpha^{-1} R^{-1} F^{-1} \Lambda P_{\Lambda} .
$$

Later we will see that $-T^{\prime}=P_{M}$, as it will be conjugate to a new canonical coordinate $M$. Following this procedure to the end, we may then find the form of the new coordinate $M(t, r)$, also as a function of $t$ and $r$. First, we need to know the form of $F$ as a function of the canonical pair $\Lambda, R$. For that, we replace back into Eq. (223) the definition of $T^{\prime}$, giving

$$
F=\left(\frac{R^{\prime}}{\Lambda}\right)^{2}-\left(\frac{P_{\Lambda}}{\alpha R}\right)^{2} .
$$

Equating this form of $F$ with Eq. (219), we obtain

$$
M=\frac{1}{2} \alpha R\left(\frac{\alpha^{2}}{16} R^{2}+\frac{\alpha^{2} R^{2}}{16}-F\right),
$$

where $F$ is given in Eq. (229). We thus have found the form of the new canonical coordinate, $M$. It is now a straightforward calculation to determine the Poisson bracket of this variable with $P_{M}=-T^{\prime}$ and see that they are conjugate, thus making Eq. (228) the conjugate momentum of $M$, i.e.,

$$
P_{M}=\alpha^{-1} R^{-1} F^{-1} \Lambda P_{\Lambda} .
$$

The other natural transformation has been given, namely the one that relates $P_{\Gamma}$ with $Q$ through $P_{\Gamma}=Q$. As $M, Q$ is also a natural choice for canonical coordinate, being another physical parameter of the solution (24). The one coordinate which remains to be found is $P_{Q}$, the conjugate momentum to the charge. It is also necessary to find out the other new canonical variable which commutes with $M, P_{M}$, and $Q$, and which guarantees, with its conjugate momentum, that the transformation from $\Lambda, R, \Gamma$, to $M, Q$, and the new variable is canonical. Immediately is it seen that $R$ commutes with $M, P_{M}$, and $Q$. It is then a candidate. It remains to be seen whether $P_{R}$ also commutes with $M, P_{M}$, and $Q$. As with $R$, it is straightforward to see that $P_{R}$ does not commute with $M$ and $P_{M}$, as these contain powers of $R$ in their definitions, and $\left\{R(t, r), P_{R}\left(t, r^{*}\right)\right\}=\delta\left(r-r^{*}\right)$. So rename the canonical variable $R$ as $R=\mathrm{R}$. We have then to find a new conjugate momentum to $\mathrm{R}$ which also commutes with $M, P_{M}$, and $Q$, making the transformation from $\left\{\Lambda, R, \Gamma ; P_{\Lambda}, P_{R}, P_{\Gamma}\right\} \rightarrow\left\{M, \mathrm{R}, Q ; P_{M}, P_{\mathrm{R}}, P_{Q}\right\}$ a canonical one. The way to proceed is to look at the constraint $H_{r}$, which is called in this formalism the super-momentum. This is the constraint which generates spatial diffeomorphisms in all variables. Its form, in the initial canonical coordinates, is $H_{r}=-\Lambda P_{\Lambda}^{\prime}+P_{R} R^{\prime}-\Gamma P_{\Gamma}^{\prime}$. In this formulation, $\Lambda$ is a spatial density, $R$ is a spatial scalar, and $\Gamma$ is also a spatial density. As the new variables, $M, \mathrm{R}$, and $Q$, are spatial scalars, the generator of spatial diffeomorphisms is written as $H_{r}=P_{M} M^{\prime}+P_{\mathrm{R}} \mathrm{R}^{\prime}+P_{Q} Q^{\prime}$, regardless of the particular form of the canonical coordinate transformation. It is thus equating these two expressions of the super-momentum $H_{r}$, with $M, P_{M}$, and $Q$ written as functions of $\Lambda, R, \Gamma$ and their respective momenta, that gives us one equation for the new $P_{\mathrm{R}}$ and $P_{Q}$. This means that we have two unknowns, sc. $P_{\mathrm{R}}$ and $P_{Q}$, for one equation only. This sugests that we should make the coefficients of $R^{\prime}$ and $P_{\Gamma}^{\prime}$ equal to zero independently. This results in

$$
\begin{aligned}
P_{\mathrm{R}}= & P_{R}-\frac{3 \alpha^{2}}{32} F^{-1} \Lambda P_{\Lambda} R-\frac{1}{2} R^{-1} \Lambda P_{\Lambda}+F^{-1} P_{\Lambda} R^{\prime \prime} \Lambda^{-1}-F^{-1} \Lambda^{-2} P_{\Lambda} \Lambda^{\prime} R^{\prime}+\left(R^{\prime}\right)^{2} F^{-1} \Lambda^{-1} P_{\Lambda} R^{-1} \\
& -F^{-1} \Lambda^{-1} P_{\Lambda}^{\prime} R^{\prime}+\frac{1}{2} \alpha^{-2} R^{-3} P_{\Gamma}^{2} F^{-1} \Lambda P_{\Lambda}, \\
P_{Q}= & -\Gamma-\alpha^{-2} R^{-2} F^{-1} \Lambda P_{\Lambda} P_{\Gamma} .
\end{aligned}
$$

We have now all the canonical variables of the new set determined. For completeness and future use, we write the inverse transformation for $\Lambda$ and $P_{\Lambda}$,

$$
\begin{aligned}
\Lambda & =\left(\left(\mathrm{R}^{\prime}\right)^{2} F^{-1}-P_{M}^{2} F\right)^{\frac{1}{2}} \\
P_{\Lambda} & =\alpha \mathrm{R} F P_{M}\left(\left(\mathrm{R}^{\prime}\right)^{2} F^{-1}-P_{M}^{2} F\right)^{-\frac{1}{2}} .
\end{aligned}
$$


In summary, the canonical transformations are

$$
\begin{aligned}
R= & \mathrm{R} \\
M= & \frac{1}{2} \alpha R\left(\frac{\alpha^{2}}{16} R^{2}-F\right) \\
Q= & P_{\Gamma} \\
P_{\mathrm{R}}= & P_{R}-\frac{3 \alpha^{2}}{32} F^{-1} \Lambda P_{\Lambda} R-\frac{1}{2} R^{-1} \Lambda P_{\Lambda}+F^{-1} P_{\Lambda} R^{\prime \prime} \Lambda^{-1}-F^{-1} \Lambda^{-2} P_{\Lambda} \Lambda^{\prime} R^{\prime}+\left(R^{\prime}\right)^{2} F^{-1} \Lambda^{-1} P_{\Lambda} R^{-1} \\
& -F^{-1} \Lambda^{-1} P_{\Lambda}^{\prime} R^{\prime}+\frac{1}{2} \alpha^{-2} R^{-3} P_{\Gamma}^{2} F^{-1} \Lambda P_{\Lambda}, \\
P_{M}= & \alpha^{-1} R^{-1} F^{-1} \Lambda P_{\Lambda}, \\
P_{Q}= & -\Gamma-\alpha^{-2} R^{-2} F^{-1} \Lambda P_{\Lambda} P_{\Gamma} .
\end{aligned}
$$

In order to prove that the set of equalities in expression (236) is canonical we start with the equality

$$
\begin{aligned}
P_{\Lambda} \delta \Lambda+P_{R} \delta R+P_{\Gamma} \delta \Gamma-P_{M} \delta M-P_{\mathrm{R}} \delta \mathrm{R}-P_{Q} \delta Q= & \left(\frac{1}{2} \alpha R \delta R \ln \left|\frac{\alpha R R^{\prime}+\Lambda P_{\Lambda}}{\alpha R R^{\prime}-\Lambda P_{\Lambda}}\right|\right)^{\prime}+ \\
& +\delta\left(\Gamma P_{\Gamma}+\Lambda P_{\Lambda}+\frac{1}{2} \alpha R R^{\prime} \ln \left|\frac{\alpha R R^{\prime}-\Lambda P_{\Lambda}}{\alpha R R^{\prime}+\Lambda P_{\Lambda}}\right|\right)
\end{aligned}
$$

We now integrate expression (237) in $r$, in the interval from $r=0$ to $r=\infty$. The first term on the right hand side of Eq. (237) vanishes due to the falloff conditions (see Eqs. (194)-(202) and Eqs. (204)-(212)). We then obtain the following expression

$$
\int_{0}^{\infty} d r\left(P_{\Lambda} \delta \Lambda+P_{R} \delta R+P_{\Gamma} \delta \Gamma\right)-\int_{0}^{\infty} d r\left(P_{M} \delta M+P_{\mathrm{R}} \delta \mathrm{R}+P_{Q} \delta Q\right)=\delta \omega\left[\Lambda, R, \Gamma, P_{\Lambda}, P_{\Gamma}\right]
$$

where $\delta \omega\left[\Lambda, R, \Gamma, P_{\Lambda}, P_{\Gamma}\right]$ is a well defined functional, which is also an exact form. This equality shows that the difference between the Liouville form of $\left\{R, \Lambda, \Gamma ; P_{R}, P_{\Lambda}, P_{\Gamma}\right\}$ and the Liouville form of $\left\{\mathrm{R}, M, Q ; P_{\mathrm{R}}, P_{M}, P_{Q}\right\}$ is an exact form, which implies that the transformation of variables given by the set of equations (236) is canonical.

Armed with the certainty of the canonicity of the new variables, we can write the asymptotic form of the canonical variables and of the metric function $F(t, r)$. These are, for $r \rightarrow 0$

$$
\begin{aligned}
F(t, r) & =4 R_{2}(t) \Lambda_{0}(t)^{-2} r^{2}+O\left(r^{4}\right), \\
\mathrm{R}(t, r) & =R_{0}(t)+R_{2}(t) r^{2}+O\left(r^{4}\right), \\
M(t, r) & =M_{0}(t)+M_{2}(t) r^{2}+O\left(r^{4}\right), \\
Q(t, r) & =Q_{0}(t)+Q_{2}(t) r^{2}+O\left(r^{4}\right), \\
P_{\mathrm{R}}(t, r) & =O(r), \\
P_{M}(t, r) & =O(r), \\
P_{Q}(t, r) & =O(r),
\end{aligned}
$$

with

$$
\begin{aligned}
& M_{0}(t)=\frac{1}{2} \alpha^{-1} R_{0}^{-1}(t) Q_{0}^{2}(t)+\frac{1}{32} \alpha^{3} R_{0}^{3}(t) \\
& M_{2}(t)=\frac{1}{32} \alpha R_{0}(t) R_{2}(t)\left(3 \alpha^{2} R_{0}(t)-64 R_{2}(t) \Lambda_{0}(t)^{-2}\right)+\alpha^{-1} Q_{0}(t) R_{0}^{-1}(t)\left(Q_{2}(t)-\frac{1}{2} Q_{0}(t) R_{0}^{-1}(t) R_{2}(t)\right)
\end{aligned}
$$

For $r \rightarrow \infty$, we have

$$
\begin{aligned}
F(t, r) & =\frac{\alpha^{2}}{16} r^{2}-2(\eta(t)+2 \rho(t)) r^{-1}+O^{\infty}\left(r^{-2}\right) \\
\mathrm{R}(t, r) & =r+16 \rho(t) \alpha^{-2} r^{-2}+O^{\infty}\left(r^{-3}\right) \\
M(t, r) & =M_{+}(t)+O^{\infty}\left(r^{-1}\right) \\
Q(t, r) & =Q_{+}(t)+O^{\infty}\left(r^{-1}\right) \\
P_{\mathrm{R}}(t, r) & =O^{\infty}\left(r^{-4}\right) \\
P_{M}(t, r) & =O^{\infty}\left(r^{-6}\right) \\
P_{Q}(t, r) & =O^{\infty}\left(r^{-2}\right)
\end{aligned}
$$


where $M_{+}(t)=\alpha(\eta(t)+3 \rho(t))$, as seen before in the surface terms (see Eq. (214)).

We are now almost ready to write the action with the new canonical variables. It is now necessary to determine the new Lagrange multipliers. In order to write the new constraints with the new Lagrange multipliers, we can use the identity given by the space derivative of $M$,

$$
M^{\prime}=-\Lambda^{-1}\left(R^{\prime} H+\alpha^{-1} R^{-1} P_{\Lambda}\left(H_{r}-\Gamma G\right)+\alpha^{-1} \Lambda R^{-1} P_{\Gamma} G\right)
$$

Solving for $H$ and making use of the inverse transformations of $\Lambda$ and $P_{\Lambda}$, in Eqs. (234) and (235), we get

$$
\begin{aligned}
H & =-\frac{M^{\prime} F^{-1} \mathrm{R}^{\prime}+F P_{M} P_{\mathrm{R}}+\alpha^{-1} R^{-1} F^{-1} R^{\prime} Q G}{\left(F^{-1}\left(\mathrm{R}^{\prime}\right)^{2}-F P_{M}^{2}\right)^{\frac{1}{2}}}, \\
H_{r} & =P_{M} M^{\prime}+P_{\mathrm{R}} \mathrm{R}^{\prime}+P_{Q} Q^{\prime} \\
G & =-Q^{\prime}
\end{aligned}
$$

Following Kuchař [10], the new set of constraints, totally equivalent to the old set $H(t, r)=0, H_{r}(t, r)=0$, and $G(t, r)=0$ outside the horizon, is $M^{\prime}(t, r)=0, P_{\mathrm{R}}(t, r)=0$, and $Q^{\prime}(t, r)=0$. By continuity, this also applies on the horizon, where $F(t, r)=0$. So we can say that the equivalence is valid everywhere. So, the new Hamiltonian, the total sum of the constraints, can now be written as

$$
N H+N^{r} H_{r}+\tilde{\Phi} G=N^{M} M^{\prime}+N^{\mathrm{R}} P_{\mathrm{R}}+N^{Q} Q^{\prime}
$$

In order to determine the new Lagrange multipliers, one has to write the left hand side of the previous equation, Eq. (259), and replace the constraints on that side by their expressions as functions of the new canonical coordinates, spelt out in Eqs. (256) - (258). After manipulation, one gets

$$
\begin{aligned}
N^{M} & =-\frac{N F^{-1} R^{\prime}}{\left(F^{-1}\left(\mathrm{R}^{\prime}\right)^{2}-F P_{M}^{2}\right)^{\frac{1}{2}}}+N^{r} P_{M}, \\
N^{\mathrm{R}} & =-\frac{N F P_{M}}{\left(F^{-1}\left(\mathrm{R}^{\prime}\right)^{2}-F P_{M}^{2}\right)^{\frac{1}{2}}}+N^{r} R^{\prime}, \\
N^{Q} & =\frac{\alpha^{-1} N R^{-1} F^{-1} R^{\prime} Q}{\left(F^{-1}\left(\mathrm{R}^{\prime}\right)^{2}-F P_{M}^{2}\right)^{\frac{1}{2}}}+N^{r} P_{Q}-\tilde{\Phi} .
\end{aligned}
$$

Using the inverse transformations Eqs. (234)-(235), and the identity $R=\mathrm{R}$, we can write the new multipliers as functions of the old variables

$$
\begin{aligned}
N^{M} & =-N F^{-1} R^{\prime} \Lambda^{-1}+\alpha^{-1} N^{r} F^{-1} R^{-1} \Lambda P_{\Lambda}, \\
N^{\mathrm{R}} & =-\alpha^{-1} N R^{-1} P_{\Lambda}+N^{r} R^{\prime}, \\
N^{Q} & =\alpha^{-1} N \Lambda^{-1} R^{-1} F^{-1} R^{\prime} Q-N^{r} \Gamma-\alpha^{-2} N^{r} R^{-2} F^{-1} \Lambda P_{\Lambda} P_{\Gamma}-\tilde{\Phi},
\end{aligned}
$$

allowing us to determine its asymptotic conditions from the original conditions given above. These transformations are non-singular for $r>0$. As before, for $r \rightarrow 0$,

$$
\begin{aligned}
N^{M}(t, r) & =-\frac{1}{2} N_{1}(t) \Lambda_{0}(t) R_{2}(t)^{-1}+O\left(r^{2}\right) \\
N^{\mathrm{R}}(t, r) & =O\left(r^{2}\right) \\
N^{Q}(t, r) & =\frac{1}{2} \alpha^{-1} N_{1}(t) Q_{0}(t) \Lambda_{0}(t) R_{0}^{-1}(t) R_{2}^{-1}(t)-\tilde{\Phi}_{0}+O\left(r^{2}\right)
\end{aligned}
$$

and for $r \rightarrow \infty$ we have

$$
\begin{aligned}
N^{M}(t, r) & =-\tilde{N}_{+}(t)+O^{\infty}\left(r^{-4}\right), \\
N^{\mathrm{R}}(t, r) & =O^{\infty}\left(r^{-2}\right), \\
N^{Q}(t, r) & =-\tilde{\Phi}_{+}(t)+O^{\infty}\left(r^{-1}\right) .
\end{aligned}
$$


These conditions (266)- (271) show that the transformations in Eqs. (263)-(264) are satisfactory in the case of $r \rightarrow \infty$, but not for $r \rightarrow 0$. This is due to fact that in order to fix the Lagrange multipliers for $r \rightarrow \infty$, as we are free to do, we fix $\tilde{N}_{+}(t)$, which we already do when adding the surface term

$$
-\int d t \tilde{N}_{+} M_{+}
$$

to the action, in order to obtain the equations of motion in the bulk, without surface terms. The same is true for $\tilde{\Phi}_{+}$. However, at $r=0$, we see that fixing the multiplier $N^{M}$ to values independent of the canonical variables is not the same as fixing $N_{1} \Lambda_{0}^{-1}$ to values independent of the canonical variables. The same is true of the fixation of $N^{Q}$ with respect to $\tilde{\Phi}_{0}$. We need to rewrite the multipliers $N^{M}$ and $N^{Q}$ for the asymptotic regime $r \rightarrow 0$ without affecting their behavior for $r \rightarrow \infty$. In order to proceed we have to make one assumption, which is that the expression given in asymptotic condition of $M(t, r)$, as $r \rightarrow 0$, for the term of order zero, $M_{0} \equiv \frac{1}{2} \alpha^{-1} Q_{0}(t)^{2} R_{0}^{-1}(t)+\frac{1}{32} \alpha^{3} R_{0}(t)^{3}$, defines $R_{0}$ as a function of $M_{0}$ and $Q_{0}$, where $R_{0}$ is the horizon radius function, $R_{0} \equiv R_{\mathrm{h}}\left(M_{0}, Q_{0}\right)$. Also, we assume that $M_{0}>M_{\text {crit }}\left(Q_{0}\right)=\frac{Q^{\frac{3}{2}}}{3^{\frac{3}{4}}}$. With these assumptions, we are working in the domain of the classical solutions. We can immediately obtain that the variation of $R_{0}$ is given in relation to the variations of $M_{0}$ and of $Q_{0}$ as

$$
\delta R_{0}=\left(\frac{32}{3} \alpha^{3} R_{0}^{2}-\frac{Q_{0}^{2}}{2 \alpha R_{0}^{2}}\right)^{-1}\left(\delta M_{0}-\frac{Q_{0} \delta Q_{0}}{\alpha R_{0}}\right) .
$$

This expression will be used when we derive the equations of motion from the new action. We now define the new multipliers $\tilde{N}^{M}$ and $\tilde{N}^{Q}$ as

$$
\begin{aligned}
& \tilde{N}^{M}=-N^{M}\left[(1-g)+g\left(\alpha R_{0}\right)\left(\frac{3 \alpha^{3}}{32} R_{0}^{2}-\frac{Q_{0}^{2}}{2 \alpha R_{0}^{2}}\right)^{-1}\right]^{-1}, \\
& \tilde{N}^{Q}=\tilde{N}^{M} g Q_{0}\left(\frac{3 \alpha^{3}}{32} R_{0}^{2}-\frac{Q_{0}^{2}}{2 \alpha R_{0}^{2}}\right)^{-1}-N^{Q},
\end{aligned}
$$

where $g(r)=1+O\left(r^{2}\right)$ for $r \rightarrow 0$ and $g(r)=O^{\infty}\left(r^{-5}\right)$ for $r \rightarrow \infty$. These new multipliers, functions of the old multipliers $N^{M}$ and $N^{Q}$, have as their properties for $r \rightarrow \infty$

$$
\begin{aligned}
\tilde{N}^{M}(t, r) & =\tilde{N}_{+}(t)+O^{\infty}\left(r^{-5}\right), \\
\tilde{N}^{Q}(t, r) & =\tilde{\Phi}_{+}(t)+O^{\infty}\left(r^{-1}\right),
\end{aligned}
$$

and for $r \rightarrow 0$

$$
\begin{aligned}
& \tilde{N}^{M}(t, r)=\tilde{N}_{0}^{M}(t)+O\left(r^{2}\right), \\
& \tilde{N}^{M}(t, r)=\tilde{\Phi}_{0}(t)+O\left(r^{2}\right)
\end{aligned}
$$

where $\tilde{N}_{0}^{M}$ is given by

$$
\tilde{N}_{0}^{M}=\frac{1}{2} \alpha^{-1} N_{1} R_{0}^{-1} R_{2}^{-1} \Lambda_{0}\left(-\frac{1}{2} Q_{0}^{2} \alpha^{-1} R_{0}^{-2}+\frac{3}{32} \alpha^{3} R_{0}^{2}\right) .
$$

When the constraints $M^{\prime}=0=Q^{\prime}$ hold, the last expression is

$$
\tilde{N}_{0}^{M}=N_{1} \Lambda_{0}^{-1}
$$

With this new constraint $\tilde{N}^{M}$, fixing $N_{1} \Lambda_{0}^{-1}$ at $r=0$ or fixing $\tilde{N}_{0}^{M}$ is equivalent. The same happens with fixing $\tilde{\Phi}_{0}$ or $\tilde{N}^{Q}$ at $r \rightarrow 0$. There are no problems with $N^{\mathrm{R}}$, which is left as determined in Eq. (261). The new action is now written as the sum of $S_{\Sigma}$, the bulk action, and $S_{\partial \Sigma}$, the surface action,

$$
\begin{aligned}
S\left[M, \mathrm{R}, \Gamma, P_{M}, P_{\mathrm{R}}, P_{\Gamma} ; \tilde{N}^{M}, N^{\mathrm{R}}, \tilde{N}^{Q}\right] & =\int d t \int_{0}^{\infty} d r\left\{P_{M} \dot{M}+P_{\mathrm{R}} \dot{\mathrm{R}}+P_{Q} \dot{Q}+\tilde{N}^{Q} Q^{\prime}-N^{\mathrm{R}} P_{\mathrm{R}}\right. \\
& \left.+\tilde{N}^{M}\left[(1-g) M^{\prime}+g\left(\frac{3 \alpha^{2}}{16} R_{0}^{2}-\frac{Q_{0}^{2}}{2 \alpha R_{0}^{2}}\right)^{-1}\left(\alpha R_{0} M^{\prime}-Q_{0} Q^{\prime}\right)\right]\right\} \\
& +\int d t\left(\frac{1}{2} \alpha R_{0}^{2} \tilde{N}_{0}^{M}-\tilde{N}_{+} M_{+}+\tilde{\Phi}_{0} Q_{0}-\tilde{\Phi}_{+} Q_{+}\right) .
\end{aligned}
$$


The new equations of motion are now

$$
\begin{aligned}
\dot{M} & =0, \\
\dot{\mathrm{R}} & =N^{\mathrm{R}}, \\
\dot{Q} & =0, \\
\dot{P}_{M} & =\left(N^{M}\right)^{\prime}, \\
\dot{P}_{\mathrm{R}} & =0 \\
\dot{P}_{Q} & =\left(N^{Q}\right)^{\prime}, \\
M^{\prime} & =0 \\
P_{\mathrm{R}} & =0 \\
Q^{\prime} & =0
\end{aligned}
$$

Here we understood $N^{M}$ to be a function of the new constraint $\tilde{N}^{M}$, defined through Eq. (274), and $N^{Q}$ to be a function of the new constraint $\tilde{N}^{Q}$, defined through Eq. (275). The resulting boundary terms of the variation of this new action, Eq. (282), are, first, terms proportional to $\delta M, \delta \mathrm{R}$, and $\delta Q$ on the initial and final hypersurfaces, and, second, the term

$$
\int d t\left(\frac{1}{2} \alpha R_{0}^{2} \delta \tilde{N}_{0}^{M}-M_{+} \delta \tilde{N}_{+}+Q_{0} \delta \tilde{\Phi}_{0}-Q_{+} \delta \tilde{\Phi}_{+}\right)
$$

Here we have used the expression in Eq. (273). The action in Eq. (282) yields the equations of motion, Eqs. (283)(291), provided that we fix the initial and final values of the new canonical variables and that we also fix the values of $\tilde{N}_{0}^{M}, \tilde{N}_{+}, \tilde{\Phi}_{0}$, and $\tilde{\Phi}_{+}$. Thanks to the redefinition of both the Lagrange multipliers, from $N^{M}$ to $\tilde{N}^{M}$ and from $N^{Q}$ to $\tilde{N}^{Q}$, the fixation of those quantities, $\tilde{N}_{0}^{M}, \tilde{N}_{+}, \tilde{\Phi}_{0}$, and $\tilde{\Phi}_{+}$has the same meaning it had before the canonical transformations and the redefinition of $N^{M}$ and $N^{Q}$. This same meaning is guaranteed through the use of our gauge freedom to choose the multipliers, and at the same time not fixing the boundary variations independently of the choice of Lagrange multipliers, which in turn allow us to have a well defined variational principle for the action.

\section{Hamiltonian reduction}

We now solve the constraints in order to reduce to the true dynamical degrees of freedom. The equations of motion (283)-(291) allow us to write $M$ and $Q$ as independent functions of the radial coordinate $r$,

$$
\begin{aligned}
M(t, r) & =\mathbf{m}(t), \\
Q(t, r) & =\mathbf{q}(t) .
\end{aligned}
$$

The reduced action, with the constraints and Eqs. (293)-(294) taken into account, is

$$
S\left[\mathbf{m}, \mathbf{p}_{\mathbf{m}}, \mathbf{q}, \mathbf{p}_{\mathbf{q}} ; \tilde{N}_{0}^{M}, \tilde{N}_{+}, \tilde{\Phi}_{0}, \tilde{\Phi}_{+}\right]=\int d t \mathbf{p}_{\mathbf{m}} \dot{\mathbf{m}}+\mathbf{p}_{\mathbf{q}} \dot{\mathbf{q}}-\mathbf{h}
$$

where

$$
\begin{aligned}
\mathbf{p}_{\mathbf{m}} & =\int_{0}^{\infty} d r P_{M}, \\
\mathbf{p}_{\mathbf{q}} & =\int_{0}^{\infty} d r P_{Q},
\end{aligned}
$$

and the reduced Hamiltonian, h, is now written as

$$
\mathbf{h}(\mathbf{m}, \mathbf{q} ; t)=-\frac{1}{2} \alpha R_{\mathrm{h}}^{2} \tilde{N}_{0}^{M}+\tilde{N}_{+} \mathbf{m}+\left(\tilde{\Phi}_{+}-\tilde{\Phi}_{0}\right) \mathbf{q}
$$

with $R_{\mathrm{h}}$ being the horizon radius. We also have that $\mathbf{m}>M_{\text {crit }}(\mathbf{q})=\frac{\mathbf{q}^{\frac{3}{2}}}{3^{\frac{3}{4}}}$, according to the assumptions made in the previous subsection. Thanks to the functions $\tilde{N}_{0}^{M}(t), \tilde{N}_{+}(t), \tilde{\Phi}_{0}(t)$, and $\tilde{\Phi}_{+}(t)$ the Hamiltonian $\mathbf{h}$ is an explicitly 
time dependent function. The variational principle associated with the reduced action, Eq. (295), will fix the value of $\mathbf{m}$ and $\mathbf{q}$ on the initial and final hypersurfaces, or in the spirit of the classical analytical mechanics, the Hamiltonian principle fixes the initial and final values of the canonical coordinate. The equations of motion are

$$
\begin{aligned}
\dot{\mathbf{m}} & =0 \\
\dot{\mathbf{q}} & =0 \\
\dot{\mathbf{p}}_{\mathbf{m}} & =\alpha R_{\mathrm{h}}\left(\frac{3}{32} \alpha^{3} R_{\mathrm{h}}^{2}-\frac{\mathbf{q}^{2}}{2 \alpha R_{\mathrm{h}}^{2}}\right)^{-1} \tilde{N}_{0}^{M}-\tilde{N}_{+} \\
\dot{\mathbf{p}}_{\mathbf{q}} & =-\mathbf{q}\left(\frac{3}{32} \alpha^{3} R_{\mathrm{h}}^{2}-\frac{\mathbf{q}^{2}}{2 \alpha R_{\mathrm{h}}^{2}}\right)^{-1} \tilde{N}_{0}^{M}+\tilde{\Phi}_{0}-\tilde{\Phi}_{+}
\end{aligned}
$$

The equation of motion for $\mathbf{m}$, Eq. (299), is understood as saying that $\mathbf{m}$ is, on a classical solution, equal to the mass parameter $M$ of the solutions in Eq. (177). The same holds for $\mathbf{q}$ in Eq. (300), i.e., $\mathbf{q}$ is the charge parameter in Eqs. (177)-(179). In order to interpret equation of motion for $\mathbf{p}_{\mathbf{m}}, \mathrm{Eq}$. (301), we have to recall that from Eq. (231) one has $P_{M}=-T^{\prime}$, where $T$ is the Killing time. This, together with the definition of $\mathbf{p}_{\mathbf{m}}$, given in Eq. (296), yields

$$
\mathbf{p}_{\mathbf{m}}=T_{0}-T_{+}
$$

where $T_{0}$ is the value of the Killing time at the left end of the hypersurface of a certain $t$, and $T_{+}$is the Killing time at spatial infinity, the right end of the same hypersurface of $t$. As the hypersurface evolves in the spacetime of the black hole solutions, the right hand side of Eq. (1300) is equal to $\dot{T}_{0}-\dot{T}_{+}$. Finally, after the definition

$$
\mathbf{p}_{\mathbf{q}}=\xi_{0}-\xi_{+}
$$

obtained from to Eqs. (217), (233), and (297), Eq. (302) gives in the right hand side $\dot{\xi}_{0}-\dot{\xi}_{+}$, which is the diference of the time derivatives of the electromagnetic gauge $\xi(t, r)$ at $r=0$ and at infinity.

\section{E. Quantum theory and partition function}

Following Sec. III we need not repeat here the steps taken there in order to build the time evolution operator.

Thus the operator $K$ can now be written as

$$
K\left(\mathbf{m}, \mathbf{q} ; \mathcal{T}, \Theta, \Xi_{0}, \Xi_{+}\right)=\exp \left[-i \mathbf{m} \mathcal{T}+\frac{i}{2} \alpha R_{\mathrm{h}}^{2} \Theta-i \mathbf{q}\left(\Xi_{+}-\Xi_{0}\right)\right]
$$

The same composition law as in Sec. III holds here as well. Here again $\mathcal{T}$ is the Killing time elapsed at right spatial infinity and $\Theta$ is the boost parameter elapsed at the bifurcation circle; $\Xi_{0}$ and $\Xi_{+}$are line integrals along timelike curves of constant $r$ and constant angular variables, at $r=0$ and at infinity.

\section{F. Thermodynamics}

We can now build the partition function for this system. The path to follow is to continue the operator to imaginary time and take the trace over a complete orthogonal basis. Our classical thermodynamic situation consists of a threedimensional spherically symmetric charged black hole, asymptotically anti-de Sitter, in thermal equilibrium with a bath of Hawking radiation. Ignoring back reaction from the radiation, the geometry is described by the solutions in Eq. (177) and (179). Thus, we consider a thermodynamic ensemble in which the temperature, or more appropriately here, the inverse temperature $\beta$ is fixed. This characterizes a grand canonical ensemble, and the partition function $\mathcal{Z}(\beta, \bar{\phi})$ arises naturally in such an ensemble. To analytically continue the Lorentzian solution we put $\mathcal{T}=-i \beta$, and $\Theta-2 \pi i$, this latter choice based on the regularity of the classical Euclidean solution. We also choose $\Xi_{0}=0$ and $\Xi_{+}=i \beta \bar{\phi}$.

We arrive then at the following expression for the partition function

$$
\mathcal{Z}(\beta, \bar{\phi})=\operatorname{Tr}[\hat{K}(-i \beta,-2 \pi i, 0, i \beta \bar{\phi})]
$$


From Eq. (305) this is realized as

$$
\mathcal{Z}(\beta, \bar{\phi})=\int_{A} \mu d \mathbf{m} d \mathbf{q} \exp \left[-\beta(\mathbf{m}-\mathbf{q} \bar{\phi})+\pi \alpha R_{\mathrm{h}}^{2}\right]\langle\mathbf{m} \mid \mathbf{m}\rangle
$$

Since $\langle\mathbf{m} \mid \mathbf{m}\rangle$ is equal to $\delta(0)$, one has to regularize [307). Again, following the Louko-Whiting procedure [2]-[6], we have to regularize and normalize the operator $\hat{K}$ beforehand. This leads to

$$
\mathcal{Z}_{\text {ren }}(\beta, \bar{\phi})=\mathcal{N} \int_{A} \mu d \mathbf{m} d \mathbf{q} \exp \left[-\beta(\mathbf{m}-\mathbf{q} \bar{\phi})+\pi \alpha R_{\mathrm{h}}^{2}\right],
$$

where $\mathcal{N}$ is a normalization factor and $A$ is the domain of integration. Provided the weight factor $\mu$ is slowly varying compared to the exponential in Eq. (308), and using the fact that the horizon radius $R_{\mathrm{h}}$ is function of $\mathbf{m}$ and $\mathbf{q}$, the integral in Eq. (308) is convergent. Changing integration variables, from $\mathbf{m}$ to $R_{\mathrm{h}}$, where

$$
\mathbf{m}=\frac{1}{32} \alpha^{3} R_{\mathrm{h}}^{3}+\frac{\mathbf{q}^{2}}{2 \alpha R_{\mathrm{h}}}
$$

the integral Eq. (308) becomes

$$
\mathcal{Z}_{\mathrm{ren}}(\beta, \bar{\phi})=\mathcal{N} \int_{A^{\prime}} \widetilde{\mu} d R_{\mathrm{h}} d \mathbf{q} \exp \left(-I_{*}\right)
$$

where $A^{\prime}$ is the new domain of integration after changing variables, and the function $I_{*}\left(R_{\mathrm{h}}, \mathbf{q}\right)$, a kind of an effective action (see [16]), is written as

$$
I_{*}\left(R_{\mathrm{h}}, \mathbf{q}\right):=\frac{1}{32} \beta \alpha^{3} R_{\mathrm{h}}^{3}+\frac{\beta}{2 \alpha} \frac{\mathbf{q}^{2}}{R_{\mathrm{h}}}-\beta \bar{\phi} \mathbf{q}-\pi \alpha R_{\mathrm{h}}^{2} .
$$

The domain of integration, $A^{\prime}$, is defined by the inequalities $0 \leq R_{\mathrm{h}}$ and $\mathbf{q}^{2} \leq 3 \alpha^{4} R_{\mathrm{h}}^{4} / 16$. The new weight factor $\tilde{\mu}$ includes the Jacobian of the change of variables. Since the weight factor is slowly varying, we can estimate the integral of $\mathcal{Z}_{\text {ren }}(\beta, \bar{\phi})$ by the saddle point approximation. The critical points are given as a pair of values $\left(R_{\mathrm{h}}, \mathbf{q}\right)$

$$
\begin{aligned}
R_{\mathrm{h}}^{ \pm} & =\frac{32 \pi}{3 \beta \alpha^{2}}\left(1 \pm \sqrt{1+\frac{3}{64} \beta^{2} \alpha^{2} \bar{\phi}^{2} \pi^{-2}}\right), \\
\mathbf{q}^{ \pm} & =\alpha R_{\mathrm{h}}^{ \pm} \bar{\phi}
\end{aligned}
$$

The critical points belong to the domain of the effective action (311), and so the effective action has a null derivative at these points in Eqs. (312)-(313). As $\bar{\phi}^{2}>-\frac{3}{64} \beta^{-2} \alpha^{-2} \bar{\phi}^{-2} \pi^{2}$ there are always two critical points, given by (313)-(312). The critical point at $\left(R_{\mathrm{h}}^{-}, \mathbf{q}^{-}\right)$is outside the domain of physical interest as $R_{\mathrm{h}}^{-}<0$. The critical point at $\left(R_{\mathrm{h}}^{+}, \mathbf{q}^{+}\right)$is an global minimum. The expression (313) implies that the critical points are on the straight line defined by itself, on the plane of the arguments of the effective action (311). Then, if we replace the value of the charge as a function of $R_{\mathrm{h}}$, i. e., $\mathbf{q}\left(R_{\mathrm{h}}\right)$ as given in (313) into the effective action we obtain a one variable function of $R_{\mathrm{h}}$, written as

$$
I_{*}\left(R_{\mathrm{h}}\right)=\frac{1}{32} \beta \alpha^{3} R_{\mathrm{h}}^{3}-\frac{1}{2} \beta \alpha \bar{\phi}^{2} R_{\mathrm{h}}-\alpha \pi R_{\mathrm{h}}^{2},
$$

which is a function of $R_{\mathrm{h}}$ on the straight line defined by $\mathbf{q}=\alpha R_{\mathrm{h}} \bar{\phi}$. The zeros of this effective action on the straight line are given by

$$
\begin{aligned}
R_{\mathrm{h}}^{0} & =0 \\
R_{\mathrm{h}}^{0 \pm} & =16 \beta^{-1} \alpha^{-2} \pi\left(1 \pm \sqrt{1+\frac{1}{16} \beta^{2} \alpha^{2} \bar{\phi}^{2} \pi^{-2}}\right)
\end{aligned}
$$

Here $R_{\mathrm{h}}^{-}<0$ is outside the physical domain of interest. As $\bar{\phi}^{2}>-\frac{1}{16} \beta^{-2} \alpha^{-2} \bar{\phi}^{-2} \pi^{2}$ there are always three zeros of the function (314), which means that there are no phase transitions, as the zeros of the effective action maintain their respective hierarchy whatever the value of $\bar{\phi}$, which is the order parameter of the system. So the classical solution 
is found in the global minimum of the critical points $\left(R_{\mathrm{h}}^{+}, \mathbf{q}^{+}\right)$of the effective action $I_{*}\left(R_{\mathrm{h}}, \mathbf{q}\right)$ (Eq. (311) ). The partition function $\mathcal{Z}_{\text {ren }}(\beta, \bar{\phi})$ can now be written, through the saddle-point approximation

$$
\begin{aligned}
\mathcal{Z}_{\mathrm{ren}}(\beta, \bar{\phi}) & \approx \mathrm{P} \exp \left[-I_{*}\left(R_{\mathrm{h}}^{+}, \mathbf{q}^{+}\right)\right], \\
\ln \mathcal{Z}_{\mathrm{ren}}(\beta, \bar{\phi}) & \approx \ln \mathrm{P}-I_{*}\left(R_{\mathrm{h}}^{+}, \mathbf{q}^{+}\right) .
\end{aligned}
$$

where $\mathrm{P}$ is a slowly varying prefactor, and $I_{*}\left(R_{\mathrm{h}}^{+}, \mathbf{q}^{+}\right)$is the effective action evaluated at the global minimum critical point. By ignoring the prefactor's logarithm, which closer to $\left(R_{\mathrm{h}}^{+}, \mathbf{q}^{+}\right)$is less relevant, we are able to determine the value of $\mathbf{m}$ at the critical point, where we find that it corresponds to the value of the mass of the classical solution of the black hole given in Eq. (177). Thus, when the critical point dominates the partition function, we have that the mean energy $\langle E\rangle$ is given by

$$
\langle E\rangle=-\frac{\partial}{\partial \beta} \ln \mathcal{Z}_{\text {ren }} \approx \frac{1}{32} \alpha^{3}\left(R_{\mathrm{h}}^{+}\right)^{3}-\frac{1}{2} \alpha \bar{\phi}^{2} R_{\mathrm{h}}^{+}=\mathbf{m}^{+},
$$

where $\mathbf{m}^{+}$is obtained from Eq. (309) evaluated at $\left(R_{\mathrm{h}}^{+}, \mathbf{q}^{+}\right)$. The thermal expectation value of the charge is

$$
\langle Q\rangle=\beta^{-1} \frac{\partial}{\partial \phi} \ln \mathcal{Z}_{\text {ren }} \approx \mathbf{q}^{+},
$$

as given in Eq. (313). We now can write the temperature $\mathbf{T} \equiv \beta^{-1}$ as a function of the critical point given by $R_{\mathrm{h}}^{+}$ and $\mathbf{q}^{+}$

$$
\mathbf{T}=\frac{1}{4 \pi}\left(3 a R_{\mathrm{h}}^{+}-\frac{1}{16}\left(\mathbf{q}^{+}\right)^{2}\left(a R_{\mathrm{h}}^{+}\right)^{-3}\right)
$$

with $R_{\mathrm{h}}^{+}$explicitly written in (312), and where $\mathbf{q}^{+}$is given in Eq. (313). If we choose the maximum charge of the domain given by $\mathbf{q}^{2} \leq 3 \alpha^{4} R_{\mathrm{h}}^{4} / 16$, then we find ourselves with the extreme solution of the black hole, whose temperature, if $\mathbf{q}^{2}=3 \alpha^{4} R_{\mathrm{h}}^{4} / 16$ is replaced into (321), is equal to zero. It can be shown that $\partial \mathbf{m}^{+} / \partial \beta<0$, which through the constant $\bar{\phi}$ heat capacity $C_{\bar{\phi}}=-\beta^{2}(\partial\langle E\rangle / \partial \beta)$ tells us that the system is thermodynamically stable. Finally there is the entropy of the black hole, $S$, which is given by

$$
S=\left(1-\beta \frac{\partial}{\partial \beta}\right)\left(\ln \mathcal{Z}_{\mathrm{ren}}\right) \approx 4 \pi a R_{\mathrm{h}}^{+^{2}} .
$$

Here we see that despite the fact that the extreme solution has null temperature, it has an entropy given in (322), which is independent of the value of the charge in the domain of validity of the approximation.

\section{HAMILTONIAN THERMODYNAMICS OF A REPRESENTATIVE CHARGED DILATONIC BLACK HOLE $(\omega=-3)$}

\section{A. The metric}

For $\omega=-3$, the general metric in Eq. (21), the $\phi$ field in Eq. (6), and the vector potential (8) reduce to the following, respectively,

$$
\begin{aligned}
d s^{2} & =-\left[(a R)^{2}-2(a R)^{\frac{1}{2}} M-\frac{1}{2}(a R) Q^{2}\right] d T^{2}+\frac{d R^{2}}{(a R)^{2}-2(a R)^{\frac{1}{2}} M-\frac{1}{2}(a R) Q^{2}}+R^{2} d \varphi^{2}, \\
e^{-2 \phi} & =\frac{1}{(a R)^{\frac{1}{2}}} \\
A & =-\frac{1}{2}(a R)^{\frac{1}{2}} Q d T,
\end{aligned}
$$

with $2 M=b$ and $a=\frac{4 \sqrt{3}}{3}|\lambda|=\frac{2 \sqrt{6}}{3} l^{-1}$. In Fig. 3 the Carter-Penrose diagram of the black hole solution for the case 


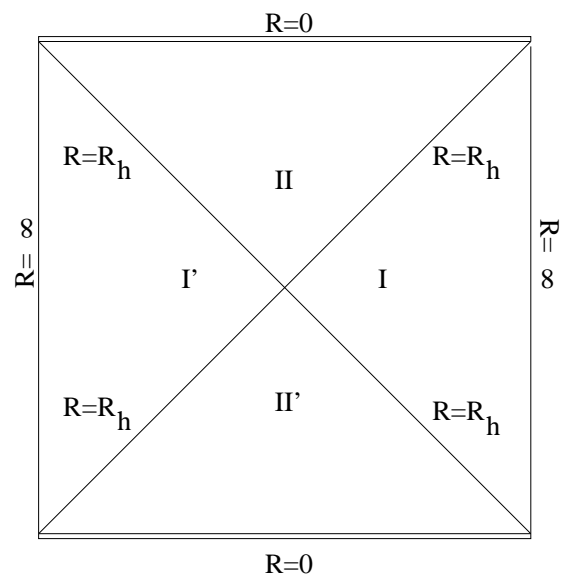

FIG. 3: The Carter-Penrose Diagram for the $\omega=-3$ case, with $M>0$.

$\omega=-3$ is shown, which is analogous to the case $\omega=0$, where again the singularity at $R=0$ is a curvature singularity, and $R=\infty$ is spatial infinity. The horizon radius is given by the largest real solution of the following equation

$$
\left(a R_{\mathrm{h}}\right)^{2}-2\left(a R_{\mathrm{h}}\right)^{\frac{1}{2}} M-\frac{1}{2}\left(a R_{\mathrm{h}}\right) Q^{2}=0 .
$$

Note that this diagram, Fig. 3, holds for static black holes with positive mass $M>0$. Here I and I' are the right and left static outer regions of the Carter-Penrose diagram, respectively, and II and II' are the future and past inner dynamical regions of the Carter-Penrose diagram. $R_{\mathrm{h}}$ is the horizon radius given in Eq. (326) and the spacelike infinity is asymptotically anti-de Sitter, both in the right and in the left regions, denoted by I and I', respectively. There are no extreme black hole solutions for $\omega=-3$ [25].

Since we are now familiar with the whole formalism, we will be briefer in this section omitting several of the details.

\section{B. Canonical formalism}

The action becomes, following Eqs. (21)-(23), and up to surface terms,

$$
\begin{aligned}
S\left[\Lambda, R, \dot{\Lambda}, \dot{R} ; N, N^{r}\right]= & \int d t \int_{0}^{\infty} d r\left\{4(a R)^{-\frac{1}{2}} N \lambda^{2} \Lambda R-(a R)^{-\frac{1}{2}} N^{-1} \dot{\Lambda} \dot{R}+\frac{1}{4}(a R)^{-\frac{1}{2}} N^{-1} \Lambda R^{-1} \dot{R}^{2}\right. \\
& +(a R)^{-\frac{1}{2}} N^{-1} \dot{R}\left(N^{r} \Lambda\right)^{\prime}+(a R)^{-\frac{1}{2}} N^{-1} N^{r} \dot{\Lambda} R^{\prime}-(a R)^{-\frac{1}{2}} N^{-1} N^{r}\left(N^{r}\right)^{\prime} \Lambda R^{\prime} \\
& -(a R)^{-\frac{1}{2}} N^{-1}\left(N^{r}\right)^{2} \Lambda^{\prime} R^{\prime}-\frac{1}{2}(a R)^{-\frac{1}{2}} N^{-1} N^{r} \Lambda R^{-1} \dot{R} R^{\prime}+\frac{1}{4}(a R)^{-\frac{1}{2}} N^{-1}\left(N^{r}\right)^{2} \Lambda R^{-1}\left(R^{\prime}\right)^{2} \\
& +\frac{1}{4}(a R)^{-\frac{1}{2}} N \Lambda^{-1} R^{-1}\left(R^{\prime}\right)^{2}-(a R)^{-\frac{1}{2}} N\left(\Lambda^{-1}\right)^{\prime} R^{\prime}-(a R)^{-\frac{1}{2}} N \Lambda^{-1} R^{\prime \prime} \\
& \left.+2 N^{-1} \Lambda^{-1}(a R)^{-\frac{1}{2}} R\left(\dot{\Gamma}-\Phi^{\prime}\right)^{2}\right\} .
\end{aligned}
$$

Depending on the situation we use three different letters containing the same information but with slightly different numerical values. Thus, $a, \lambda$, and $l$ are related by $a^{2}=\frac{16}{3} \lambda^{2}=\frac{8}{3} l^{-2}$, where $l$ is defined now as the AdS length. From the action above, we obtain the conjugate momenta

$$
\begin{aligned}
& P_{\Lambda}=-N^{-1}(a R)^{-\frac{1}{2}}\left(\dot{R}-R^{\prime} N^{r}\right) \\
& P_{R}=-N^{-1}(a R)^{-\frac{1}{2}}\left\{\dot{\Lambda}-\left(\Lambda N^{r}\right)^{\prime}-\frac{1}{2} \Lambda R^{-1}\left(\dot{R}-R^{\prime} N^{r}\right)\right\} \\
& P_{\Gamma}=4 N^{-1} \Lambda^{-1}(a R)^{-\frac{1}{2}} R\left(\dot{\Gamma}-\Phi^{\prime}\right) .
\end{aligned}
$$

By performing a Legendre transformation we obtain the Hamiltonian, which is a sum of constraints, i.e.,

$$
\mathcal{H}=N\left\{-(a R)^{\frac{1}{2}} P_{\Lambda} P_{R}+\frac{1}{4} a^{\frac{1}{2}} R^{-\frac{1}{2}} \Lambda P_{\Lambda}^{2}+(a R)^{-\frac{1}{2}}\left[\left(\Lambda^{-1}\right)^{\prime} R^{\prime}+\Lambda^{-1} R^{\prime \prime}-\frac{1}{4} \Lambda^{-1}\left(R^{\prime}\right)^{2} R^{-1}\right]\right.
$$




$$
\begin{aligned}
& \left.-4(a R)^{-\frac{1}{2}} \lambda^{2} \Lambda R+\frac{1}{8} N \Lambda R^{-1}(a R)^{\frac{1}{2}} P_{\Gamma}^{2}\right\} \\
& +N^{r}\left\{P_{R} R^{\prime}-\Lambda P_{\Lambda}^{\prime}-\Gamma P_{\Gamma}^{\prime}\right\}+\tilde{\Phi}\left\{-P_{\Gamma}^{\prime}\right\} \equiv N H+N^{r} H_{r}+\tilde{\Phi} G .
\end{aligned}
$$

We can now write the action in Hamiltonian form, which reads

$$
S\left[\Lambda, R, \Gamma, P_{\Lambda}, P_{R}, P_{\Gamma} ; N, N^{r}, \tilde{\Phi}\right]=\int d t \int_{0}^{\infty} d r\left\{P_{\Lambda} \dot{\Lambda}+P_{R} \dot{R}+P_{\Gamma} \dot{\Gamma}-N H-N^{r} H_{r}-\tilde{\Phi} G\right\},
$$

with the constraints defined in Eq. (331). From here we derive the equations of motion for the canonical variables and respective canonical momenta

$$
\begin{aligned}
\dot{\Lambda}= & -N(a R)^{\frac{1}{2}} P_{R}+\frac{1}{2} N a^{\frac{1}{2}} R^{-\frac{1}{2}} \Lambda P_{\Lambda}+\left(N^{r} \Lambda\right)^{\prime} \\
\dot{R}= & -N(a R)^{\frac{1}{2}} P_{\Lambda}+N^{r} R^{\prime} \\
\dot{\Gamma}= & \frac{1}{4} N(a R)^{\frac{1}{2}} \Lambda R^{-1} P_{\Gamma}+\left(N^{r} P_{\Gamma}\right)^{\prime}+\tilde{\Phi}^{\prime} \\
\dot{P}_{\Lambda}= & -\frac{1}{4} N a^{\frac{1}{2}} R^{-\frac{1}{2}} P_{\Lambda}^{2}-\left(N(a R)^{-\frac{1}{2}}\right)^{\prime} R^{\prime} \Lambda^{-2}-\frac{1}{4} N(a R)^{-\frac{1}{2}}\left(R^{\prime}\right)^{2} R^{-1} \Lambda^{-2}+4 N(a R)^{-\frac{1}{2}} \lambda^{2} R+N^{r} P_{\Lambda}^{\prime} \\
& -\frac{1}{8} N(a R)^{\frac{1}{2}} R^{-1} P_{\Gamma}^{2} \\
\dot{P}_{R}= & \frac{1}{2} N a^{\frac{1}{2}} R^{-\frac{1}{2}} P_{\Lambda} P_{R}+\frac{1}{8} N a^{\frac{1}{2}} R^{-\frac{3}{2}} \Lambda P_{\Lambda}^{2}-N a^{-\frac{1}{2}}\left(\Lambda^{-1}\right)^{\prime}\left(R^{-\frac{1}{2}}\right)^{\prime} \\
& +\frac{1}{4} N(a R)^{-\frac{1}{2}} \Lambda^{-1} R^{-1} R^{\prime \prime}-\left(\left(N a^{-\frac{1}{2}}\right)^{\prime} \Lambda^{-1} R^{-\frac{1}{2}}\right)^{\prime}-\frac{1}{2} N a^{-\frac{1}{2}} \Lambda^{-1}\left(R^{-\frac{1}{2}}\right)^{\prime \prime} \\
& +2 N a^{-\frac{1}{2}} \lambda^{2} \Lambda R^{-\frac{1}{2}}+\left(N^{r} P_{R}\right)^{\prime}+\frac{1}{16} N \Lambda(a R)^{\frac{1}{2}} R^{-2} P_{\Gamma}^{2}, \\
\dot{P}_{\Gamma}= & N^{r} P_{\Gamma}^{\prime} .
\end{aligned}
$$

For a correct variational principle to be applied, we have to find out what surface terms are left over from the variation performed, with the purpose of deriving the equations of motion in Eqs. (333)-(338). These surface terms are,

$$
\begin{aligned}
\text { Surface terms }= & N(a R)^{-\frac{1}{2}} R^{\prime} \Lambda^{-2} \delta \Lambda-N(a R)^{-\frac{1}{2}} \Lambda^{-1} \delta R^{\prime}+\left((a R)^{-\frac{1}{2}}\right)^{\prime} \Lambda^{-1} \delta R+\frac{1}{2}(a R)^{-\frac{1}{2}} \Lambda^{-1} R^{-1} R^{\prime} \delta R \\
& -N^{r} P_{R} \delta R+N^{r} \Lambda \delta P_{\Lambda}+N^{r} \Gamma \delta P_{\Gamma}+\left.\tilde{\Phi} \delta P_{\Gamma}\right|_{0} ^{\infty} .
\end{aligned}
$$

In order to know the form of (339) for $r \rightarrow 0$, we assume

$$
\begin{aligned}
\Lambda(t, r) & =\Lambda_{0}+O\left(r^{2}\right), \\
R(t, r) & =R_{0}+R_{2} r^{2}+O\left(r^{4}\right), \\
P_{\Lambda}(t, r) & =O\left(r^{3}\right) \\
P_{R}(t, r) & =O(r), \\
N(t, r) & =N_{1}(t) r+O\left(r^{3}\right) \\
N^{r}(t, r) & =O\left(r^{3}\right), \\
\Gamma(t, r) & =O(r), \\
P_{\Gamma}(t, r) & =Q_{0}+Q_{2} r^{2}+O\left(r^{4}\right), \\
\tilde{\Phi}(t, r) & =\tilde{\Phi}_{0}(t)+O\left(r^{2}\right) .
\end{aligned}
$$

The surface terms become

$$
\text { Surface terms }\left.\right|_{r=0}=-N_{1}(a R)^{-\frac{1}{2}} \Lambda_{0}^{-1} \delta R_{0}-\tilde{\Phi}_{0} \delta Q_{0} .
$$

The asymptotic conditions for $r \rightarrow \infty$ are assumed as

$$
\Lambda(t, r)=\frac{\sqrt{6}}{4} l r^{-1}+2^{\frac{5}{4}} l^{\frac{5}{2}} \eta(t) r^{-\frac{5}{2}}+O^{\infty}\left(r^{-3}\right),
$$




$$
\begin{aligned}
R(t, r) & =r+2^{\frac{3}{4}} l^{\frac{3}{2}} \rho(t) r^{-\frac{1}{2}}+O^{\infty}\left(r^{-1}\right), \\
P_{\Lambda}(t, r) & =O^{\infty}\left(r^{-2}\right), \\
P_{R}(t, r) & =O^{\infty}\left(r^{-4}\right), \\
N(t, r) & =R(t, r)^{\prime} \Lambda(t, r)^{-1}\left(\tilde{N}_{+}(t)+O^{\infty}\left(r^{-5}\right)\right), \\
N^{r}(t, r) & =O^{\infty}\left(r^{-2}\right), \\
\Gamma(t, r) & =O^{\infty}\left(r^{-2}\right), \\
P_{\Gamma}(t, r) & =Q_{+}(t)+O^{\infty}\left(r^{-1}\right), \\
\tilde{\Phi}(t, r) & =\tilde{\Phi}_{+}(t)+O^{\infty}\left(r^{-1}\right) .
\end{aligned}
$$

where $l$ is the AdS length. The surface terms, Eq. (339), for $r \rightarrow \infty$, are written as

$$
\text { Surface terms }\left.\right|_{r \rightarrow \infty}=\tilde{N}_{+} \delta M_{+}+\tilde{\Phi}_{+} \delta Q_{+},
$$

where

$$
M_{+}(t)=2^{5} 3^{-\frac{5}{4}} \eta(t)+2^{2} 3^{\frac{1}{2}} \rho(t) .
$$

Here we pay attention to the fact that we can define the above quantity $M_{+}$, in Eq. (360), because we do as explained in subsection $\llbracket \mathrm{A}$ when $\omega<-\frac{3}{2}$ and absorb the divergence in the definition of $M$. This means that the $M_{+}$above in Eq. (360) is in fact related to the mass plus the divergence, defined above as $M\left(R_{*}\right)$, see Eq. (201) and below in subsection $\mathrm{VC}$, The surface term added to (332) is then

$$
S_{\partial \Sigma}\left[\Lambda, R, Q_{0}, Q_{+} ; N, \tilde{\Phi}_{0}, \tilde{\Phi}_{+}\right]=\int d t\left(2 a^{-1}\left(a R_{0}\right)^{\frac{1}{2}} N_{1} \Lambda_{0}^{-1}-\tilde{N}_{+} M_{+}+\tilde{\Phi}_{0} Q_{0}-\tilde{\Phi}_{+} Q_{+}\right) .
$$

With this surface term added we obtain a well defined variational principle. What remains after variation of the total action, Eq. (332) and Eq. (361), is

$$
\int d t\left(2 a^{-1}\left(a R_{0}\right)^{\frac{1}{2}} \delta\left(N_{1} \Lambda_{0}^{-1}\right)-M_{+} \delta \tilde{N}_{+}+Q_{0} \delta \tilde{\Phi}_{0}-Q_{+} \delta \tilde{\Phi}_{+}\right) .
$$

The surface terms coming from the variation of the total action disappear as the result of the fixation of $N_{1} \Lambda_{0}^{-1}$ on the horizon, $r=0$, and of $\tilde{N}_{+}(t)$ at infinity, $r \rightarrow \infty$, with the same happening respectively to $\tilde{\Phi}_{0}$ at $r=0$ and to $\tilde{\Phi}_{+}$ at $r \rightarrow \infty$.

\section{Reconstruction, canonical transformation, and action}

Repeating the steps of the two previous sections, we now give the main results concerning $\omega=-3$. The vector potential is

$$
A=-\frac{1}{2}(a R)^{\frac{1}{2}} Q d T+\xi
$$

where there is the function $\xi=\xi(t, r)$. Expanding for $(t, r)$ we find

$$
A=\left(-\frac{1}{2}(a R)^{\frac{1}{2}} Q T^{\prime}+\xi^{\prime}\right) d r+\left(-\frac{1}{2}(a R)^{\frac{1}{2}} Q \dot{T}+\dot{\xi}\right) d t,
$$

out of which we infer that

$$
P_{\Gamma}=Q .
$$

The metric function is given by the expression

$$
F=(a R)^{2}-2(a R)^{\frac{1}{2}} M-\frac{1}{2}(a R) P_{\Gamma}^{2} .
$$

The Killing time $T=T(t, r)$ is a function of $(t, r)$, where we find that

$$
-T^{\prime}=F^{-1} \Lambda P_{\Lambda}(a R)^{\frac{1}{2}},
$$


which is equal to minus the conjugate momentum of the new variable $M$, or $P_{M}=P_{M}(t, r) \equiv-T^{\prime}(t, r)$. With the help of Eq. (367) we find $F=F(t, r)$ as a function of the canonical variables,

$$
F=\left(R^{\prime}\right)^{2} \Lambda^{-2}-a R P_{\Lambda}^{2} .
$$

Summing up, the canonical transformations are

$$
\begin{aligned}
R= & \mathrm{R} \\
M= & \frac{1}{2}(a R)^{-\frac{1}{2}}\left((a R)^{2}-\frac{1}{2}(a R) P_{\Gamma}^{2}-F\right), \\
Q= & P_{\Gamma}, \\
P_{\mathrm{R}}= & P_{R}-F^{-1} R^{-1} \Lambda^{-2}\left[R R^{\prime} \Lambda P_{\Lambda}^{\prime}+\frac{1}{2}\left(R^{\prime}\right)^{2} P_{\Lambda} \Lambda-R R^{\prime \prime} \Lambda P_{\Lambda}+R R^{\prime} \Lambda^{\prime} P_{\Lambda}\right] \\
& +\frac{1}{4} R^{-1} \Lambda P_{\Lambda}-4 \lambda^{2} F^{-1} P_{\Lambda} R \Lambda+\frac{1}{8} a F^{-1} \Lambda P_{\Lambda} P_{\Gamma}^{2}, \\
P_{M}= & F^{-1} \Lambda P_{\Lambda}(a R)^{\frac{1}{2}} \\
P_{Q}= & -\Gamma+\frac{1}{2}(a R) \Lambda P_{\Lambda} P_{\Gamma} .
\end{aligned}
$$

In addition, the relevant inverse transformations back to the old variable $\Lambda$ and respective conjugate momentum $P_{\Lambda}$ are

$$
\begin{aligned}
\Lambda & =\left(F^{-1}\left(R^{\prime}\right)^{2}-F P_{M}^{2}\right)^{\frac{1}{2}}, \\
P_{\Lambda} & =(a R)^{-\frac{1}{2}} F P_{M}\left(F^{-1}\left(R^{\prime}\right)^{2}-F P_{M}^{2}\right)^{-\frac{1}{2}} .
\end{aligned}
$$

We have to show that this transformation is canonical. This requires using the identity

$$
\begin{aligned}
P_{\Lambda} \delta \Lambda+P_{R} \delta R+P_{\Gamma} \delta \Gamma-P_{M} \delta M-P_{\mathrm{R}} \delta \mathrm{R}-P_{Q} \delta Q & =\left(\frac{1}{2}(a R)^{-\frac{1}{2}} \delta R \ln \left|\frac{(a R)^{-\frac{1}{2}} R^{\prime}+\Lambda P_{\Lambda}}{(a R)^{-\frac{1}{2}} R^{\prime}-\Lambda P_{\Lambda}}\right|\right)^{\prime}+ \\
& +\delta\left(\Gamma P_{\Gamma}+\Lambda P_{\Lambda}+\frac{1}{2}(a R)^{-\frac{1}{2}} R^{\prime} \ln \left|\frac{(a R)^{-\frac{1}{2}} R^{\prime}-\Lambda P_{\Lambda}}{(a R)^{-\frac{1}{2}} R^{\prime}+\Lambda P_{\Lambda}}\right|\right)(372
\end{aligned}
$$

We now integrate Eq. (372), in $r$, from $r=0$ to $r=\infty$. The first term on the right hand side of Eq. (372) vanishes due to the falloff conditions, Eqs. (340)-(348) and Eqs. (350)-(358). We obtain then the following expression

$$
\int_{0}^{\infty} d r\left(P_{\Lambda} \delta \Lambda+P_{R} \delta R+P_{\Gamma} \delta \Gamma\right)-\int_{0}^{\infty} d r\left(P_{M} \delta M+P_{\mathrm{R}} \delta \mathrm{R}+P_{Q} \delta Q\right)=\delta \omega\left[\Lambda, R, \Gamma, P_{\Lambda}, P_{\Gamma}\right],
$$

where $\delta \omega\left[\Lambda, R, \Gamma, P_{\Lambda}, P_{\Gamma}\right]$ is a well defined functional, which is also an exact form. As above, this equality shows that the difference between the Liouville form of $\left\{R, \Lambda, \Gamma ; P_{R}, P_{\Lambda}, P_{\Gamma}\right\}$ and the Liouville form of $\left\{\mathrm{R}, M, Q ; P_{\mathrm{R}}, P_{M}, P_{Q}\right\}$ is an exact form, which implies that the set of transformations (369) is canonical.

With this result, we write the asymptotic conditions of the new canonical coordinates for $r \rightarrow 0$

$$
\begin{aligned}
F(t, r) & =4 R_{2}^{2} \Lambda_{0}^{-2} r^{2}+O\left(r^{4}\right), \\
\mathrm{R}(t, r) & =R_{0}+R_{2} r^{2}+O\left(r^{4}\right), \\
M(t, r) & =M_{0}+M_{2} r^{2}+O\left(r^{4}\right), \\
Q(t, r) & =Q_{0}+Q_{2} r^{2}+O\left(r^{4}\right), \\
P_{\mathrm{R}}(t, r) & =O(r), \\
P_{M}(t, r) & =O(r), \\
P_{Q}(t, r) & =O(r) .
\end{aligned}
$$

where we have

$$
\begin{aligned}
& M_{0}=\frac{1}{2}\left(a R_{0}\right)^{-\frac{1}{2}}\left(\left(a R_{0}\right)^{2}-\frac{1}{2}\left(a R_{0}\right) Q_{0}^{2}\right), \\
& M_{2}=-\frac{\left[-\frac{1}{2}\left(a R_{0}\right) Q_{0}^{2}+\left(a R_{0}\right)^{2}\right] R_{2}}{4 R_{0}\left(a R_{0}\right)^{\frac{1}{2}}}+\frac{2 a^{2} R_{0}^{2} R_{2}^{2}-\frac{1}{2}\left[2\left(a R_{0}\right) Q_{0} Q_{2}+a Q_{0}^{2} R_{2}\right]-4 R_{2}^{2} \Lambda_{0}^{-2}}{2\left(a R_{0}\right)^{\frac{1}{2}}},
\end{aligned}
$$


and $a=l^{-1} \sqrt{8 / 3}$. For $r \rightarrow \infty$, we have

$$
\begin{aligned}
F(t, r) & =\frac{4}{3} l^{-2} r^{2}-\frac{16}{3}\left(\frac{\sqrt{2} l^{-1}}{6}\right)^{\frac{1}{2}}\left(8 \eta(t)+3^{\frac{1}{2}} \rho(t)\right) r^{\frac{1}{2}}+O^{\infty}\left(r^{0}\right) \\
\mathrm{R}(t, r) & =r+\left(\frac{\sqrt{2}}{2} l^{-1}\right)^{-\frac{3}{2}} \rho(t) r^{-\frac{1}{2}}+O^{\infty}\left(r^{-1}\right) \\
Q(t, r) & =Q_{+}(t)+O^{\infty}\left(r^{-1}\right) \\
M(t, r) & =M_{+}(t)+O^{\infty}\left(r^{-\frac{1}{2}}\right) \\
P_{\mathrm{R}}(t, r) & =O^{\infty}\left(r^{-4}\right) \\
P_{M}(t, r) & =O^{\infty}\left(r^{-\frac{9}{5}}\right) \\
P_{Q}(t, r) & =O^{\infty}\left(r^{-2}\right) .
\end{aligned}
$$

where $M_{+}(t)$ is defined in Eq. (360).

We now write the future constraint $M^{\prime}$ as a function of the older constraints

$$
M^{\prime}=-\Lambda^{-1}\left(R^{\prime} H+(a R)^{\frac{1}{2}} P_{\Lambda}\left(H_{r}-\Gamma G\right)-\frac{1}{2}(a R)^{\frac{1}{2}} \Lambda P_{\Gamma} G\right) .
$$

Using the inverse transformations of $\Lambda$ and $P_{\Lambda}$ in Eqs. (370) and (371), we obtain the same form for the old constraints as functions of the new variables

$$
\begin{aligned}
H & =-\frac{M^{\prime} F^{-1} \mathrm{R}^{\prime}+F P_{M} P_{\mathrm{R}}-\frac{1}{2}(a R)^{\frac{1}{2}} Q Q^{\prime} F^{-1} R^{\prime}}{\left(F^{-1}\left(\mathrm{R}^{\prime}\right)^{2}-F P_{M}^{2}\right)^{\frac{1}{2}}}, \\
H_{r} & =P_{M} M^{\prime}+P_{\mathrm{R}} \mathrm{R}^{\prime}+P_{Q} Q^{\prime} \\
G & =-Q^{\prime} .
\end{aligned}
$$

The new Hamiltonian, the total sum of the constraints, can now be written as

$$
N H+N^{r} H_{r}+\tilde{\Phi} G=N^{M} M^{\prime}+N^{\mathrm{R}} P_{\mathrm{R}}+N^{Q} Q^{\prime} .
$$

The new multipliers are, using Eqs. (391)-(394),

$$
\begin{aligned}
N^{M} & =-\frac{N F^{-1} R^{\prime}}{\left(F^{-1}\left(\mathrm{R}^{\prime}\right)^{2}-F P_{M}^{2}\right)^{\frac{1}{2}}}+N^{r} P_{M}, \\
N^{\mathrm{R}} & =-\frac{N F P_{M}}{\left(F^{-1}\left(\mathrm{R}^{\prime}\right)^{2}-F P_{M}^{2}\right)^{\frac{1}{2}}}+N^{r} R^{\prime}, \\
N^{Q} & =-\frac{1}{2} \frac{N(a R)^{\frac{1}{2}} F^{-1} R^{\prime} Q}{\left(F^{-1}\left(\mathrm{R}^{\prime}\right)^{2}-F P_{M}^{2}\right)^{\frac{1}{2}}}+N^{r} P_{Q}-\tilde{\Phi} .
\end{aligned}
$$

Using the inverse transformations Eqs. (370)-( functions of the old variables

$$
\begin{aligned}
N^{M} & =-N F^{-1} R^{\prime} \Lambda^{-1}+N^{r} F^{-1} \Lambda P_{\Lambda}(a R)^{\frac{1}{2}}, \\
N^{\mathrm{R}} & =-N P_{\Lambda}(a R)^{\frac{1}{2}}+N^{r} R^{\prime}, \\
N^{Q} & =-\frac{1}{2} N(a R)^{\frac{1}{2}} F^{-1} R^{\prime} \Lambda^{-1} P_{\Gamma}-N^{r} \Gamma+\frac{1}{2} N^{r}(a R) \Lambda P_{\Lambda} P_{\Gamma}-\tilde{\Phi} .
\end{aligned}
$$

For $r \rightarrow 0$ we have,

$$
\begin{aligned}
& N^{M}(t, r)=-\frac{1}{2} N_{1}(t) \Lambda_{0} R_{2}^{-1}+O\left(r^{2}\right) \\
& N^{\mathrm{R}}(t, r)=O\left(r^{4}\right) \\
& N^{Q}(t, r)=-\frac{1}{4} Q_{0} R_{2}^{-1}\left(a R_{0}\right)^{\frac{1}{2}} N_{1} \Lambda_{0}-\tilde{\Phi}_{0}+O\left(r^{2}\right) .
\end{aligned}
$$


and for $r \rightarrow \infty$ we have

$$
\begin{aligned}
N^{M}(t, r) & =-\tilde{N}_{+}(t)+O^{\infty}\left(r^{-2}\right), \\
N^{\mathrm{R}}(t, r) & =O^{\infty}\left(r^{-\frac{1}{2}}\right), \\
N^{Q}(t, r) & =-\tilde{\Phi}_{+}(t)+O^{\infty}\left(r^{-1}\right) .
\end{aligned}
$$

Again, for $r \rightarrow 0$, fixing $N^{M}(t, r)$, which means fixing $N_{1} \Lambda_{0} R_{2}^{-1}$, is not equivalent to fixing $N_{1} \Lambda_{0}^{-1}$. It is thus necessary to rewrite $N^{M}$ for $r \rightarrow 0$. The same is true of fixing $N^{Q}(t, r)$ when $r \rightarrow 0$. In this case it is not the same if one fixes $-\frac{1}{4} Q_{0} R_{2}^{-1}\left(a R_{0}\right)^{\frac{1}{2}} N_{1} \Lambda_{0}-\tilde{\Phi}_{0}$ or just $\tilde{\Phi}_{0}$. So, assuming $M_{0}$ as a function of $R_{0}$ and $Q_{0}$ allows one to define the horizon radius $R_{0} \equiv R_{\mathrm{h}}\left(M_{0}, Q_{0}\right)$. We are thus working in the domain where $M_{0}>M_{\text {crit }}\left(Q_{0}\right)=\left(a^{\frac{3}{2}}-1\right) \frac{Q_{0}^{3}}{12 \sqrt{6}}$, the domain of the classical black hole solution. The variation of $R_{0}$ is given in terms of the variations of $M_{0}$ and of $Q_{0}$ in the expression

$$
\delta R_{0}=\left(\frac{3}{4} a(a R)^{\frac{1}{2}}-\frac{1}{8} a\left(a R_{0}\right)^{-\frac{1}{2}} Q_{0}^{2}\right)^{-1}\left(\delta M_{0}+\frac{1}{2}\left(a R_{0}\right)^{\frac{1}{2}} Q_{0} \delta Q_{0}\right)
$$

The new multiplier $\tilde{N}^{M}$ is obtained from the old $N^{M}$ as

$$
\begin{aligned}
& \tilde{N}^{M}=-N^{M}\left[(1-g)+\left(a R_{0}\right)^{-\frac{1}{2}} g\left(\frac{3}{4} a\left(a R_{0}\right)^{\frac{1}{2}}-\frac{1}{8} a\left(a R_{0}\right)^{-\frac{1}{2}} Q_{0}^{2}\right)^{-1}\right]^{-1}, \\
& \tilde{N}^{Q}=-\frac{1}{2} \tilde{N}^{M} g Q_{0}\left(\frac{3}{4} a\left(a R_{0}\right)^{\frac{1}{2}}-\frac{1}{8} a\left(a R_{0}\right)^{-\frac{1}{2}} Q_{0}^{2}\right)^{-1}-N^{Q},
\end{aligned}
$$

where $a=\frac{4 \sqrt{3}}{3}|\lambda|=l^{-1} \sqrt{8 / 3}$, and $g(r)=1+O\left(r^{2}\right)$ for $r \rightarrow 0$ and $g(r)=O^{\infty}\left(r^{-5}\right)$ for $r \rightarrow \infty$. This new multiplier, function of the old multiplier, $\tilde{N}^{M}$, has as its properties for $r \rightarrow \infty$

$$
\begin{aligned}
\tilde{N}^{M}(t, r) & =\tilde{N}_{+}(t)+O^{\infty}\left(r^{-2}\right), \\
\tilde{N}^{Q}(t, r) & =\tilde{\Phi}_{+}(t)+O^{\infty}\left(r^{-1}\right),
\end{aligned}
$$

and as its properties for $r \rightarrow 0$

$$
\begin{aligned}
\tilde{N}^{M}(t, r) & =\tilde{N}_{0}^{M}(t)+O\left(r^{2}\right) \\
\tilde{N}^{M}(t, r) & =\tilde{\Phi}_{0}(t)+O\left(r^{2}\right)
\end{aligned}
$$

where $\tilde{N}_{0}^{M}$ is given by

$$
\begin{array}{cl}
\tilde{N}_{0}^{M} & =\quad N_{1} \Lambda_{0} R_{2}^{-1}\left(\frac{3}{8} a^{2} R_{0}-\frac{3}{48} a Q_{0}^{2}\right) \\
M^{\prime}=0=Q^{\prime} & N_{1} \Lambda_{0}^{-1} .
\end{array}
$$

With this new constraint $\tilde{N}^{M}$, fixing $N_{1} \Lambda_{0}^{-1}$ or fixing $\tilde{N}_{0}^{M}$ is equivalent, and the same happens with $\tilde{N}^{Q}$, where fixing $\tilde{\Phi}_{0}$ is fixing the multiplier $\tilde{N}^{Q}$ in the limit $r \rightarrow 0$. There are no problems with $N^{\mathrm{R}}$, which is left as determined in Eq. (399).

The new action is then, summing both the bulk and the surface terms,

$$
\begin{aligned}
S\left[M, \mathrm{R}, Q, P_{M}, P_{\mathrm{R}}, P_{Q} ; \tilde{N}^{M}, N^{\mathrm{R}}, N^{Q}\right]= & \int d t \int_{0}^{\infty} d r\left\{P_{M} \dot{M}+P_{\mathrm{R}} \dot{\mathrm{R}}+P_{Q} \dot{Q}+N^{Q} Q^{\prime}-N^{\mathrm{R}} P_{\mathrm{R}}\right. \\
& +\tilde{N}^{M}\left[(1-g) M^{\prime}+g\left(\frac{3}{4} a(a R)^{\frac{1}{2}}-\frac{1}{8} a\left(a R_{0}\right)^{-\frac{1}{2}} Q_{0}^{2}\right)^{-1}\right. \\
& {\left.\left.\left[\left(a R_{0}\right)^{-\frac{1}{2}} M^{\prime}+Q_{0} Q^{\prime}\right]\right]\right\}+ } \\
& \int d t\left(2 a^{-1}\left(a R_{0}\right)^{\frac{1}{2}} \tilde{N}_{0}^{M}-\tilde{N}_{+} M_{+}+\tilde{\Phi}_{0} Q_{0}-\tilde{\Phi}_{+} Q_{+}\right) .
\end{aligned}
$$


The new equations of motion are now

$$
\begin{aligned}
\dot{M} & =0, \\
\dot{\mathrm{R}} & =N^{\mathrm{R}}, \\
\dot{Q} & =0, \\
\dot{P}_{M} & =\left(N^{M}\right)^{\prime}, \\
\dot{P}_{\mathrm{R}} & =0, \\
\dot{P}_{Q} & =\left(N^{Q}\right)^{\prime}, \\
M^{\prime} & =0, \\
P_{\mathrm{R}} & =0, \\
Q^{\prime} & =0 .
\end{aligned}
$$

Here we understood $N^{M}$ to be a function of the new constraint, defined through Eq. (408). The resulting boundary terms of the variation of this new action, Eq. (416), are, first, terms proportional to $\delta M, \delta \mathrm{R}$ and $\delta Q$ on the initial and final hypersurfaces, and, second, the term $\int d t\left(2 a^{-1}\left(a R_{0}\right)^{\frac{1}{2}} \delta \tilde{N}_{0}^{M}-M_{+} \delta \tilde{N}_{+}+Q_{0} \delta \tilde{\Phi}_{0}-Q_{+} \delta \tilde{\Phi}_{+}\right)$. Here we used the expression in Eq. (407). The action in Eq. (416) yields the equations of motion, Eqs. (417)-(425), provided that we fix the initial and final values of the new canonical variables and that we also fix both the values of $\tilde{N}_{0}^{M}$ and $\tilde{N}_{+}$, and $\tilde{\Phi}_{0}$ and $\tilde{\Phi}_{+}$. Thanks to the redefinition of the Lagrange multiplier, from $N^{M}$ to $\tilde{N}^{M}$, and from $N^{Q}$ to $\tilde{N}^{Q}$ the fixation of those quantities, $\tilde{N}_{0}^{M}$ and $\tilde{N}_{+}$, and $\tilde{\Phi}_{0}$ and $\tilde{\Phi}_{+}$, has the same meaning it had before the canonical transformations and the redefinition of $N^{M}$ and $N^{Q}$. This same meaning is guaranteed through the use of our gauge freedom to choose the multipliers, and at the same time not fixing the boundary variations independently of the choice of Lagrange multipliers, which in turn allow us to have a well defined variational principle for the action.

\section{Hamiltonian reduction}

We now solve the constraints in order to reduce to the true dynamical degrees of freedom. The equations of motion, Eqs. (417)-(425), allow us to write $M$ and $Q$ as independent functions of the radial coordinate $r$

$$
\begin{aligned}
M(t, r) & =\mathbf{m}(t), \\
Q(t, r) & =\mathbf{q}(t) .
\end{aligned}
$$

The reduced action, with the constraints and Eq (427) taken into account, is

$$
S\left[\mathbf{m}, \mathbf{q}, \mathbf{p}_{\mathbf{m}}, \mathbf{p}_{\mathbf{q}} ; \tilde{N}_{0}^{M}, \tilde{N}_{+}, \tilde{\Phi}_{0}, \tilde{\Phi}_{+}\right]=\int d t \mathbf{p}_{\mathbf{m}} \dot{\mathbf{m}}+\mathbf{p}_{\mathbf{q}} \dot{\mathbf{q}}-\mathbf{h},
$$

where

$$
\begin{gathered}
\mathbf{p}_{\mathbf{m}}=\int_{0}^{\infty} d r P_{M} \\
\mathbf{p}_{\mathbf{q}}=\int_{0}^{\infty} d r P_{Q},
\end{gathered}
$$

and the reduced Hamiltonian, $\mathbf{h}$, is now written as

$$
\mathbf{h}(\mathbf{m}, \mathbf{q} ; t)=-2 a^{-1}\left(a R_{\mathrm{h}}\right)^{\frac{1}{2}} \tilde{N}_{0}^{M}+\tilde{N}_{+} \mathbf{m}+\left(\tilde{\Phi}_{+}-\tilde{\Phi}_{0}\right) \mathbf{q},
$$

with $R_{\mathrm{h}}$ being the horizon radius. We also have that $\mathbf{m}>M_{\text {crit }}(\mathbf{q})$. The equations of motion are then

$$
\begin{aligned}
\dot{\mathbf{m}} & =0 \\
\dot{\mathbf{q}} & =0 \\
\dot{\mathbf{p}}_{\mathbf{m}} & =\left(a R_{\mathrm{h}}\right)^{-\frac{1}{2}}\left(\frac{3}{4} a\left(a R_{\mathrm{h}}\right)^{\frac{1}{2}}-\frac{1}{8} a\left(a R_{\mathrm{h}}\right)^{-\frac{1}{2}} \mathbf{q}^{2}\right)^{-1}-\tilde{N}_{+}, \\
\dot{\mathbf{p}}_{\mathbf{q}} & =\frac{1}{2} \mathbf{q}\left(\frac{3}{4} a\left(a R_{\mathrm{h}}\right)^{\frac{1}{2}}-\frac{1}{8} a\left(a R_{\mathrm{h}}\right)^{-\frac{1}{2}} \mathbf{q}^{2}\right)^{-1}+\tilde{\Phi}_{0}-\tilde{\Phi}_{+} .
\end{aligned}
$$


Here $\mathbf{m}$ and $\mathbf{q}$ are equal to the mass parameter $M$ and the charge parameter $Q$ of the classical solution in Eq. (323). The third equation of motion, Eq. (434), describes the time evolution of the difference of the Killing times on the horizon and at infinity, due to $\mathbf{p}_{\mathbf{m}}=T_{0}-T_{+}$and Eq. (429). The last equation, Eq. (435), gives the time evolution of $\mathbf{p}_{\mathbf{q}}$ as the difference between the time derivatives of the electromagnetic gauges $\xi(t, r)$ on the horizon and at infinity due to $\mathbf{p}_{\mathbf{m}}=\xi_{0}-\xi_{+}$and Eq. (430).

\section{E. Quantum theory and partition function}

The steps developed in Sections III and IV can be readily used here. So we do not spell out the corresponding calculations in detail. Thus, obtained as above, the time evolution operator is

$$
K\left(\mathbf{m}, \mathbf{q} ; \mathcal{T}, \Theta, \Xi_{0}, \Xi_{+}\right)=\exp \left[-i \mathbf{m} \mathcal{T}+2 i a^{-1}\left(a R_{\mathrm{h}}\right)^{\frac{1}{2}} \Theta-i \mathbf{q}\left(\Xi_{+}-\Xi_{0}\right)\right]
$$

We can now develop the thermodynamic analysis as in Sections III and IV.

\section{F. Thermodynamics}

We can now build the partition function for this system, with the Hamiltonian given in Eq. (431). The steps are the same as was the case with $\omega=\infty$ and $\omega=0$. The thermodynamic ensemble is also the grand canonical ensemble. Again, with $\mathcal{T}=-i \beta, \Theta=-2 \pi i, \Xi_{0}=0$, and $\Xi_{+}=i \beta \bar{\phi}$, we write the general form of the partition function as

$$
\mathcal{Z}=\operatorname{Tr}[\hat{K}(-i \beta,-2 \pi i, 0, i \beta \bar{\phi})]
$$

This is realized as

$$
\mathcal{Z}_{\mathrm{ren}}(\beta, \bar{\phi})=\mathcal{N} \int_{A^{\prime}} \widetilde{\mu} d R_{\mathrm{h}} d \mathbf{q} \exp \left(-I_{*}\right)
$$

where $\mathcal{N}$ is a normalization factor, $A^{\prime}$ is the domain of integration given by $0 \leq R_{\mathrm{h}}$ and $\mathbf{q}^{2} \leq 6 a R_{\mathrm{h}}$, and the function $I_{*}\left(R_{\mathrm{h}}, \mathbf{q}\right)$, a kind of an effective action [16]. $I_{*}\left(R_{\mathrm{h}}, \mathbf{q}\right)$, is written as

$$
I_{*}\left(R_{\mathrm{h}}, \mathbf{q}\right):=\frac{\beta}{2}\left(a R_{\mathrm{h}}\right)^{\frac{3}{2}}-\left(\frac{\beta}{4} a^{\frac{1}{2}} \mathbf{q}+4 \pi a^{-\frac{1}{2}}\right) R_{\mathrm{h}}^{\frac{1}{2}}-\mathbf{q} \bar{\phi} \beta .
$$

Its critical points are at

$$
\begin{aligned}
R_{\mathrm{h}}^{ \pm} & =\frac{4}{3} \pi \beta^{-1} a^{-2}\left(1 \pm \sqrt{1+\frac{3}{8} \beta^{2} a^{2} \pi^{-2} \bar{\phi}^{2}}\right) \\
\mathbf{q}^{ \pm} & =-2 \bar{\phi}\left(a R_{\mathrm{h}}^{ \pm}\right)^{-\frac{1}{2}}
\end{aligned}
$$

Given that $\bar{\phi}^{2}>-\frac{8}{3} \beta^{-2} a^{-2} \pi^{2}$ there are always two critical points, but only $R_{\mathrm{h}}^{+}$is physical as it is positive, $R_{\mathrm{h}}^{+}>0$. The other critical point is negative, $R_{\mathrm{h}}^{-}<0$. Determining the zeros of the action (439) when Eq. (441) holds, we get

$$
R_{\mathrm{h}}^{0 \pm}=4 \pi \beta^{-1} a^{-2}\left(1 \pm \sqrt{1-\frac{1}{8} \beta^{2} a^{2} \pi^{-2} \bar{\phi}^{2}}\right) .
$$

Now, for different values of $\bar{\phi}^{2}$ we may have different signs for the action (439) evaluated at the physical critical point $R_{\mathrm{h}}^{+}$. So, for $\bar{\phi}^{2}>8 \beta^{-2} a^{-2} \pi^{2}$ there are no zeros and $I_{*}\left(R_{\mathrm{h}}^{+}\right)>0$, where we take $\mathbf{q}^{+}=\mathbf{q}^{+}\left(R_{\mathrm{h}}^{+}\right)$to be a function of the critical point radius $R_{\mathrm{h}}^{+}$. If $\bar{\phi}^{2}=8 \beta^{-2} a^{-2} \pi^{2}$ then the effective action (439) has one zero only, at $R_{\mathrm{h}}^{+}$, i. e., $I_{*}\left(R_{\mathrm{h}}^{+}\right)=0$. Finally, if $\bar{\phi}^{2}<8 \beta^{-2} a^{-2} \pi^{2}$ then we have two zeros, given in Eq. (442), and $I_{*}\left(R_{\mathrm{h}}^{+}\right)<0$. Then, despite the variation of the sign of the effective action at the physical critical point $\left(R_{\mathrm{h}}^{+}, \mathbf{q}^{+}\right)$, there are no new minima when the order parameter $\bar{\phi}$ is changed across the border given by $\bar{\phi}^{2}=8 \beta^{-2} a^{-2} \pi^{2}$, which means that there are no phase transitions, as the classical phase corresponds always to the global minimum. We can now derive the basic thermodynamic results, 
as long as the approximation for the saddle point is valid, which means that we have to work in the neighborhood of the classical solution (323), where the critical point dominates. The expected value of the energy $E$ is

$$
\langle E\rangle=\left(-\frac{\partial}{\partial \beta}+\beta^{-1} \bar{\phi} \frac{\partial}{\partial \bar{\phi}}\right) \ln \mathcal{Z}_{\text {ren }} \approx \frac{1}{2}\left(a R_{\mathrm{h}}^{+}\right)^{\frac{3}{2}}-\frac{1}{4}\left(a R_{\mathrm{h}}^{+}\right)^{\frac{1}{2}} \mathbf{q}^{2}=\mathbf{m}^{+} .
$$

The charge expectation value is

$$
\langle Q\rangle=\beta^{-1} \frac{\partial}{\partial \bar{\phi}} \ln \mathcal{Z}_{\text {ren }} \approx \mathbf{q}^{+}
$$

Here we have the temperature $\mathbf{T} \equiv \beta^{-1}$ given by

$$
\mathbf{T}=\frac{1}{4 \pi}\left(\frac{3}{2} a^{2} R_{\mathrm{h}}^{+}-\frac{1}{2} a\left(\mathbf{q}^{+}\right)^{2}\right) .
$$

It can be shown that the derivative of $\mathbf{m}^{+}(\beta)$ with respect to $\beta$ is negative, which means that the heat capacity is positive, i. e., $C_{\bar{\phi}}=-\beta^{2}\left(\frac{\partial\langle E\rangle}{\partial \beta}\right)>0$. The system is thus stable. Finally the entropy is given by

$$
S=\left(1-\beta \frac{\partial}{\partial \beta}\right)\left(\ln \mathcal{Z}_{\mathrm{ren}}(\beta)\right) \approx 4 \pi \sqrt{a^{-1} R_{\mathrm{h}}^{+}}
$$

The result recovers the entropy for the three-dimensional charged dilatonic black hole with $\omega=-3$ (see also [28]).

\section{CONCLUSIONS}

We have extended the work done in [1] by adding a Maxwell term to the three-dimensional Brans-Dicke action, and thus, have continued Louko and collaborators' program [2, 3, 4, 4, 6, 6] (see also [ [8, 9])) of studying the thermodynamic properties through Hamiltonian methods of black holes in several theories in different dimensions. Specifically we have calculated the thermodynamic properties of black hole solutions with asymptotics that allow a well formulated Hamiltonian formalism in three-dimensional Brans-Dicke-Maxwell gravity. Only certain values of the Brans-Dicke parameter $\omega$ are allowed in this juncture. The corresponding theories are general relativity, i.e., $\omega \rightarrow \pm \infty$, a dimensionally reduced cylindrical charged four-dimensional general relativity theory, i.e., $\omega=0$, and a theory representing a class of charged dilaton-gravity theories, with a typical $\omega$ given by $\omega=-3$, all of these coupled to a Maxwell field. We have shown that for these theories, with well defined asymptotics for geometric and electromagnetic variables, the formalism fits well. It was possible to have a well defined electric field for all the values of $\omega$ studied. As in [1] several modifications were needed. First, in the powers of the fall-off conditions, and second due to the presence of the scalar field, which was reflected in the fact that it changed the powers of the radial $R$ coordinate, to name a few. Of course, the addition of electromagnetism also introduced a new pair of canonical variables, which added a good deal of complexity to the calculations. Namely, the treatment of the mass term when electromagnetic interaction is included is not trivial at all in this Hamiltonian formalism in three dimensions, since there is a divergent asymptotic behavior of the mass $M$. Within the Hamiltonian formalism we solved this problem by introducing a renormalized mass. This redefinition of the mass fits perfectly into the formalism for the $\omega \rightarrow \pm \infty$ (i.e., general relativity), it is not needed in the $\omega=0$ case, and conforms to the $\omega=-3$ case. Although, in order to build a three-dimensional Lorentzian Hamiltonian theory with electric charge, these modifications had to be made, a quantum theory and a statistical description of the systems in question in a grand canonical ensemble were derived, and the corresponding thermodynamics, with precise values for the entropy of the black holes studied, was established.

\section{Acknowledgments}

GASD is supported by grant SFRH/BD/2003 from FCT. This work was partially funded by Fundação para a Ciência e Tecnologia (FCT) - Portugal, through project POCI/FP/63943/2005. 
[1] G. A. S. Dias and J. P. S. Lemos, "Hamiltonian thermodynamics of three-dimensional dilatonic black holes", Phys. Rev. D 78, 044010 (2008).

[2] J. Louko and B. F. Whiting, Phys. Rev. D 51, 5583 (1995).

[3] S. Bose, J. Louko, L. Parker, and Y. Peleg, Phys. Rev. D 53, 5708 (1996).

[4] J. Louko and S. N. Winters-Hilt, Phys. Rev. D 54, 2647 (1996).

[5] J. Louko, J. Z. Simon, and S. N. Winters-Hilt, Phys. Rev. D 553525 (1997).

[6] D. R. Brill, J. Louko, and P. Peldan, Phys. Rev. D 563600 (1997).

[7] S. Bose, L. Parker, and Y. Peleg, Phys. Rev. D 56, 987 (1997).

[8] G. Kunstatter, R. Petryk, and S. Shelemy, Phys. Rev. D 57, 3537 (1998).

[9] A. J. M. Medved and G. Kunstatter, Phys. Rev. D 59, 104005 (1999).

[10] K. V. Kuchař, Phys. Rev. D 50, 3961 (1994).

[11] R. Arnowitt, S. Deser, and C. W. Misner, in Gravitation: an introduction to current research, edited by L. Witten (Wiley, New York, 1962), Chap. 7 gr-qc/0405109].

[12] T. Regge and C. Teitelboim, Ann. Phys. 88, 286 (1974).

[13] S. W. Hawking, Commun. Math. Phys. 43, 199 (1975).

[14] J. D. Bekenstein, Phys. Rev. D 9, 3292 (1974).

[15] S. W. Hawking, in General Relativity: An Einstein Centenary Survey, edited by S. W. Hawking and W. Israel (Cambridge University Press, Cambridge, England, 1979).

[16] J. W. York, Phys. Rev. D 33, 2092 (1986).

[17] H. W. Braden, J. D. Brown, B. F. Whiting, and J. W. York, Phys. Rev. D 42, 3376 (1990).

[18] O. B. Zaslavskii, Phys. Lett. A 152, 463 (1991).

[19] C. S. Peça and J. P. S. Lemos, Phys. Rev. D 59, 124007 (1999).

[20] M. Bañados, C. Teitelboim, and J. Zanelli, Phys. Rev. Lett. 69, 1849 (1992).

[21] J. P. S. Lemos, Phys Lett. B 353, 46 (1995).

[22] P. M. Sá, A. Kleber, and J. P. S. Lemos, Class. Quantum Grav. 13, 125 (1996).

[23] C. Martínez, C. Teitelboim, and J. Zanelli, Phys. Rev. D 61, 104013 (2000).

[24] J. P. S. Lemos and V. T. Zanchin, Phys. Rev. D 54, 3840 (1996).

[25] O. J. C. Dias and J. P. S. Lemos, Phys. Rev. D 64, 064001 (2001).

[26] O. B. Zaslavskii, Class. Quant. Grav. 11, L33 (1994).

[27] S. Carlip, Class. Quantum Grav. 12, 2853 (1995).

[28] M. Natsuume and T. Okamura, Phys. Rev. D 62, 064027 (2000). 